\title{
Solving Models of Controlled Dynamic Planar Rigid-Body Systems with Frictional Contact
}

\author{
Aaron Greenfield, Uluc Saranli and Alfred A. Rizzi \\ Robotics Institute, Carnegie Mellon University \\ Pittsburgh, PA 15213, USA
}

\begin{abstract}
Effective modeling and control of multi-body systems interacting with their environment through frictional contact remains a challenging problem. In this paper, we address the planar version of this problem by developing a general method to compute the instantaneous dynamic solution for planar rigid-bodies interacting with their environment through Coulomb frictional contacts. The resulting analytic forward solution is represented in piece-wise linear form, which admits tractable inversion for implementing behavioral control. We address the inherent problem of ambiguity in the resulting model (both between and within a particular linear model) by resorting to enumeration techniques and solve for the complete collection of possible model solutions in the presence of both contact constraints and additional task-specific linear constraints. We illustrate the application of these techniques by developing a controller to reliably achieve the dynamic self-righting of a hexapod robot.
\end{abstract}

Keywords: Rigid body dynamics, contact modeling, dynamic flipping, static indeterminacy, dynamic indeterminacy, Coulomb friction, RHex robot

\section{INTRODUCTION}

Effective operation of robotic systems in the real world critically depends on their reliable interaction with the environment through both intentionally and unintentionally established frictional contacts. Robust manipulation of either the robot or the environment through such interactions, especially for tasks which depend explicitly on accurate control of contact forces, remains a challenging problem for the robotics community. The primary difficulty arises from complexities associated with each of the variety of model choices available for describing interactions between two objects that are in contact. In particular, choices regarding contact compliance, frictional models and treatment of collisions each have significant impact on the efficiency, accuracy and tractability of the end product. Models that incorporate additional dynamic states lead to difficulties in constructing and implementing model based control strategies both because of added computational complexity, as well as the larger range of required sensing modalities. For example, using a penetration model for contact necessitates measurement (e.g. through force sensors) or estimation (e.g. through surface stiffness models) of contact forces and velocities in order to effect control. In this paper, we eschew these more complex approaches, and instead adopt a class of models that presume rigid objects and Coulomb friction at contact points. It is our conjecture that the resulting models, while still presenting some computational difficulties due to non-linearity and ambiguity, represent an effective and tractable basis for the design of reliable behavioral control systems for contact tasks. The main contribution of this paper is a technique and an associated algorithm which computes the instantaneous dynamic response (the unknown accelerations and reaction forces) for controlled multi-rigid-body systems subject to Coulomb frictional contacts.

The proposed algorithm makes it possible to predict the range of possible instantaneous body accelerations and contact forces that might result from the application of a specific control input at a specific point in the state space (configuration and velocity) of the system. Our approach is to 
construct a dynamic response function that is a piecewise linear but possibly ambiguous forward model for the dynamics of the system, which can be locally inverted to aid in the generation of control strategies. In building this function, we also rigorously characterize well-known problems associated with rigid object models involving frictional contacts, such as multiplicity and non-existence of solutions. Consequently, we are able to understand when and how the underlying models fail, allowing controllers to avoid difficult settings or to recognize when they are unavoidable. Specifically, in the presence of possible ambiguities, it becomes possible to design controllers that can either attempt to avoid inputs that result in uncertain behavior, or choose controls that maximize performance in the worst case. The end result is a well informed control system capable of regulating physical interaction with the environment to produce desired behavior.

In order to illustrate the practical application of our approach for computing the dynamics response function, we present a controller design for the self-righting of RHex, an autonomous hexapod robot capable of operating outdoors on rough and highly broken terrain [29]. The motivation for addressing this problem and basic principles for the construction of model-based flipping controllers were previously introduced in [30]. In this paper, we develop a new controller which is based on the same motivations, but is capable of significantly extending the domain of validity and robustness of the earlier "blind" controller. Most notably, we are able to eliminate all of the previous empirically motivated assumptions about the progression of particular contact modes throughout behavior execution. However, due to the computational and sensory limitations of our current experimental platform, we are only able to provide simulation studies as a comparative characterization of the new controller. Nevertheless, we present qualitative evidence to establish the accuracy of our simulated model with respect to earlier experiments on RHex in an effort to validate the simulation environment used for characterization.

Even though we choose the flipping task for RHex as our primary application domain in this paper, the pre- sented techniques are equally applicable to problems such as climbing with frictional contact (e.g., for snake-like or spider-like robots). Characteristic features of these platforms, such as the large number of degrees of freedom and their reliance on frictional contact for various forms of locomotion, result in highly flexible and minimally invasive exploration capabilities [7]. These very same features also make the analysis and control of climbing behaviors for such systems more challenging. An analytic exploration of these challenges for similar climbing systems has been reported in [5] and [22]. We believe the general method proposed in this paper represent a step toward the deployment of robust controllers for such settings as well.

The paper is organized as follows. In Section II, we outline related research in the field of rigid-body dynamics and control with Coulomb friction as well as dynamic manipulation for legged robots. In Section III, we introduce our algorithm for calculating the dynamic response of planar multi-rigid-body systems under frictional contact, including a characterization of potential multiplicity of solutions. In Section IV, we apply our technique to the problem of flipping with the RHex hexapod robot. Finally, in Section $\mathrm{V}$ we provide a conclusion and outline future directions for our research.

\section{Related ResearCh}

Modeling and analysis of rigid bodies subject to frictional contact constraints has received some attention in the robotics literature. Rajan, Burridge and Schwartz [28] and Erdmann [9] are among the first robotics researchers to explore inconsistencies and ambiguities arising from combining rigid-body models with Coulomb friction. In [28] the authors consider the dynamics of a planar rigid body in contact with one or two walls. The space of applied wrenches is partitioned into regions that correspond to particular contact modes and overlaps between these regions reveal solution ambiguities. Erdmann [9] gives an extensive characterization of solutions for the motion of a planar rigid body under an externally applied wrench and one contact point. The author characterizes inconsistency and ambiguity of solutions through the relation of friction cones to 
an obstacle in the configuration space, further generalizing this result to multi-contact solutions for a single rigid body.

Modern work in this area [2], [17], [20], [25], [31], [32] largely focuses on modeling multi-rigid-body system dynamics under friction using the Linear Complementarity Problem (LCP) framework. Among others, a class of formulations exemplified by Pfeiffer and Glocker [25] expresses LCP constraints of multi-rigid-body systems as a function of forces and accelerations in the system. Our approach in this paper adopts this general framework, although we do not incorporate explicit models of impact such as Newton and Poisson models, or consider tangential impacts as presented in [25] that can serve to resolve frictional inconsistency. Trinkle et al. [32] extend the LCP framework formulated as a function of contact forces and accelerations to three dimensional systems through a linearization of the friction cone. The authors present existence and uniqueness conditions for solutions, under the assumptions that all coefficients of friction are small and system jacobians have full column rank.

In contrast to formulations of contact constraints as a function of forces and accelerations, complementarity conditions can also be posed as a function of velocities and contact impulses through a time discretization of the system dynamic response. These methods are claimed to be capable of more cleanly addressing Painlevé type problems, where no non-impact solution exists, through a more natural and uniform treatment of impacts. For example, Anitescu and Potra [2] formulate a "time-stepping" LCP formulation wherein they are able to guarantee the existence of solutions in all cases, even though solution multiplicity still remains as an issue. The advantages of such timestepping formalisms are also discussed in Stewart's work [31].

It is only recently that the problem of control in the context of rigid-body dynamics with frictional contact started receiving more attention in the literature. Balkcom and Trinkle [3] consider ambiguities between non-fixed contact modes and generalize the notion of strong stability, posed earlier by [23] in the context of fixturing. They present an algorithm capable of determining the cone of permissi- ble wrenches to yield a particular contact mode between two slow-moving planar rigid bodies, based on polyhedral convex cone operations developed by [12]. The algorithm presented is used to design a controller for a workpiece insertion task. Notable limitations of this work include the assumption of negligible velocities and a single rigid body as well as the requirement that the task be expressible solely in terms of a desired contact mode. Nevertheless, the methods introduced in the present paper are largely motivated by their work.

In a more recent line of research, Peng, Anitescu, and Akella [24] formulate the problem of optimal control under frictional contact as a mathematical program with complementarity constraints (MPCC) and consider multi-robot coordination in the plane as their application domain. Considerable further development in solving MPCC by consideration of their elastic mode is presented in the work by Anitescu [1]. Further research in the area of control under contact constraints includes work by Huang and McClamrock [14], which considers optimal control of dynamical systems subject to complementarity constraints but neglects friction and focuses primarily on impacts. Work by Prattichizzo and Bicchi [26] characterizes the stability, controllabillity, and observability of frictional systems by introducing lumped compliance at the contacts and replacing unilateral constraints with bilateral constraints to prevent contacts from separating or sliding. Finally, Brogliato explores control problems under friction in the general context of control of complementary systems and poses a series of open questions pertaining to the controllability and stabilizability of such systems [6].

Our application domain of robotic self-righting closely relates to the literature on dynamic behaviors for legged robots. Raibert's work on dynamically stable hopping robots [27] was influential in the development of various other systems capable of performing dynamical maneuvers such as biped gymnastics [13] and brachiating robots [21]. However, in most of these cases, models of environment interaction through contact have been simplified using assumptions of infinite friction ("sticky" feet) or specific mode sequences. In contrast, the problem of dynamic self- 
righting considered in this paper critically relies on more accurate models of ground interactions and pronounces many of the advantages for our general modeling tool for controlled dynamics of multi-rigid-body systems with frictional contacts.

Most mobile robots designed to operate in unstructured environments avoid the necessity to perform self-righting maneuvers by adopting morphologies which are symmetric with respect to the horizontal plane [19]. There have only been a few robots which are explicitly capable of performing such maneuvers [10], [11], [33], but their use of special kinematic structures (for example, long extension arms or reconfigurable wheels) is not suitable for RHex's limited actuation affordance and morphology [29]. Consequently, the dynamic flipping controllers first introduced in [30], and further extended in this paper to more robustly handle models of frictional contact represent a novel approach in performing dynamic maneuvers with legged robots.

\section{Controlled Dynamics of Systems With Frictional CONTACTS}

In this paper, we focus on developing analytic, predictive models for planar systems that are sufficiently simple to admit tractable inversion for the purposes of control. To this end, we adopt the rigid-body model under Coulomb frictional contacts, whose solutions are in piecewise linear forms that are suitable for controller designs based on analytic model inversion. Even though more complex models based on structural and contact compliance promise to address some of the well-known inconsistency and ambiguity problems associated with such models, their nonlinearity and computational complexity often impairs their utility in the design of inverse model-based controllers.

In multi-rigid-body systems subject to frictional contacts, the unknown dynamic quantities are $\ddot{\mathbf{q}}$ and $\mathbf{f}$, the generalized accelerations and contact reaction forces of the system, respectively. Ideally, we would like to be able to compute the corresponding forward model as a dynamic response function in the general form

$$
\left[\begin{array}{c}
\ddot{\mathbf{q}} \\
\mathbf{f}
\end{array}\right]=\mathbf{g}(\mathbf{q}, \dot{\mathbf{q}}, \tau)
$$

For unconstrained mechanical systems, the calculation of this function involves only linear operations such as matrix inversion and the resulting solution is always linear in the control inputs $\tau$. On the other hand, computing the same function for a mechanical system subject to Coulomb friction constraints is more involved, as the instantaneous dynamic response is no longer linear, but rather piecewise linear in the control inputs. Furthermore, potential multiplicity or nonexistence of solutions for certain control inputs gives rise to complications arising from the coupling of algebraic contact constraints with the equations of motion. In particular, the overall model is globally not a proper "dynamical system" and there are regions in the control input space where it is impossible to specify a unique predictive model that satisfies all the contact and rigidity constraints. Nevertheless, for designing model based controllers, characterization and avoidance of such regions turns out to be a sufficient and effective method.

In light of these observations, we adopt the slightly more general form of the dynamic response function (1), yielding

$$
\left[\begin{array}{c}
\ddot{\mathbf{q}} \\
\mathbf{f}
\end{array}\right]=\mathbf{g}(\mathbf{q}, \dot{\mathbf{q}}, \tau, \mathbf{w})
$$

where the vector $\mathbf{w}$ accounts for solution ambiguities. We further partition the domain of this function into a number of subsets, each of which correspond to a particular contact mode and yields a linear relation between $(\ddot{\mathbf{q}}, \mathbf{f})$ and $(\tau, \mathbf{w})$ for a given system state $(\mathbf{q}, \dot{\mathbf{q}})$. A more formal definition of this function together with an algorithm for its computation will be detailed in subsequent sections.

Before we proceed further, we will find it useful to introduce the simple actuated two-link system shown in Figure 1. This system features a single actuated elbow, $\gamma$, and has three possible contact points at the elbow $\left(\mathbf{p}_{2}\right)$ and the endpoints of the links ( $\mathbf{p}_{1}$ and $\mathbf{p}_{3}$ ). We focus particularly on the immobilization problem for this system, which also turns out to be a useful component of climbing controllers for snake-like robots. In each of the following subsections, we illustrate the corresponding concepts through their application to this simple example. 


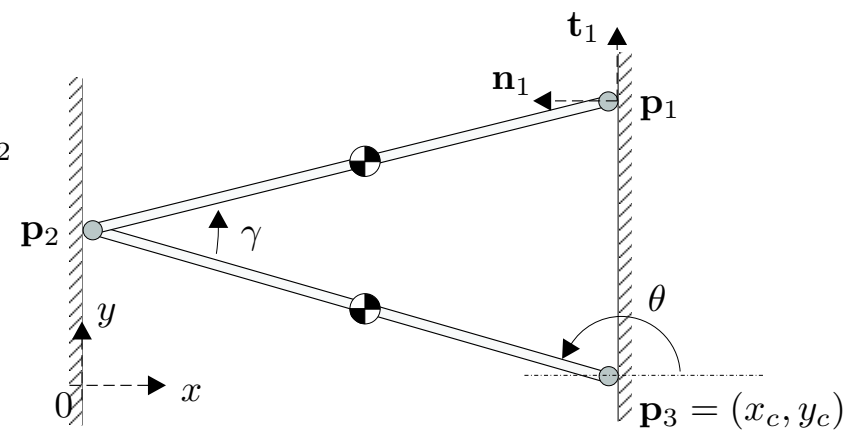

Fig. 1. An actuated two-link mechanism. Three contact points are at $\mathbf{p}_{1}, \mathbf{p}_{2}$ and $\mathbf{p}_{3}$ with contact frames $\left(\mathbf{t}_{i}, \mathbf{n}_{i}\right)$ located at each. The configuration of the robot is determined by the location of $\mathbf{p}_{3}$ and the two angles $\theta$ and $\gamma$.

\section{A. Modeling of System Dynamics}

Our algorithm presumes the availability of a Lagrangian formulation of the system dynamics, with generalized coordinates $\mathbf{q} \in \mathbb{R}^{q}$ providing a local minimal representation of the configuration of the system when unconstrained by contact. We assume that there are $k$ contact points and each has a local inertial coordinate frame $X_{i}:=\left(\mathbf{p}_{i}, \mathbf{t}_{i}, \mathbf{n}_{i}\right)$ with origin located at $\mathbf{p}_{i}$, defined such that $\mathbf{n}_{i}$ is normal to the contact surface and outward from the environment, and $\left(\mathbf{t}_{i}, \mathbf{n}_{i}\right)$ forms a right-handed coordinate system.

For a uniform treatment of contact constraints and reaction forces, we express the position of each contact point on the mechanism in the corresponding contact frame $X_{i}$, through the kinematic map ${ }^{1}$

$$
\mathbf{x}=\mathbf{h}(\mathbf{q})
$$

where we have $\mathbf{x}:=\left(x_{\mathbf{n}_{1}}, x_{\mathbf{t}_{1}}, \ldots, x_{\mathbf{n}_{k}}, x_{\mathbf{t}_{k}}\right) \in R^{2 k}$. These kinematics also yield the contact Jacobian matrix $\mathbf{J}:=D_{\mathbf{q}} \mathbf{h}$ that relates velocities and accelerations of contact points of the mechanism to the generalized coordinates through the relations

$$
\begin{aligned}
\dot{\mathbf{x}} & =\mathbf{J} \dot{\mathbf{q}} \\
\ddot{\mathbf{x}} & =\mathbf{J} \ddot{\mathbf{q}}+\dot{\mathbf{J}} \dot{\mathbf{q}} .
\end{aligned}
$$

Dynamical equations governing the motion of the system

\footnotetext{
${ }^{1}$ Our definition of the kinematic map assumes that the evolution of contact points on the robot surface (e.g in the case of rolling contact) are explicitly incorporated. A similar approach is detailed in [25].
}

take the form

$$
\mathbf{M}(\mathbf{q}) \ddot{\mathbf{q}}+\mathbf{C}(\mathbf{q}, \dot{\mathbf{q}}) \dot{\mathbf{q}}+\mathbf{g}(\mathbf{q})=\tau+\mathbf{J}^{T} \mathbf{f},
$$

where $\tau \in \mathbb{R}^{t}$ and $\mathbf{f}=\left(f_{\mathbf{n}_{1}}, f_{\mathbf{t}_{1}}, \ldots, f_{\mathbf{n}_{k}}, f_{\mathbf{t}_{k}}\right) \in \mathbb{R}^{2 k}$ denote known external control inputs and initially unknown contact reaction forces respectively; the latter being expressed in local contact coordinates.

Applied to the two-link example of Figure 1, we define the generalized coordinates as $\mathbf{q}_{\mathbf{e}}:=\left(x_{c}, y_{c}, \theta, \gamma\right)$ and the contact coordinates as $\mathbf{x}_{\mathbf{e}}:=\left(x_{\mathbf{n}_{1}}, x_{\mathbf{t}_{1}}, x_{\mathbf{n}_{2}}, x_{\mathbf{t}_{2}}, x_{\mathbf{n}_{3}}, x_{\mathbf{t}_{3}}\right)^{2}$. The contact jacobian $\mathbf{J}_{\mathbf{e}} \in \mathbb{R}^{6 \times 4}$ of (3) is easily constructed from the forward kinematics, while the remaining system matrices, $\mathbf{M}_{\mathbf{e}}, \mathbf{C}_{\mathbf{e}}, \mathbf{g}_{\mathbf{e}}$ of (5) can be computed from straightforward application of Lagrange's equations.

\section{B. Contact Modes and Constraints on Dynamics}

In the presence of frictional contacts, the dynamic equations of (5) alone are not sufficient to model system behavior. Associated with each contact, there are also complementarity constraints on corresponding accelerations and reaction forces induced by the rigid-body with Coulomb friction contact model. By definition, a complementarity constraint between two variables requires that both variables are non-negative and at least one of them is zero. For example the normal contact acceleration and the normal contact reaction force for all contacts are constrained to be complementary.

Complementarity conditions arising from non-penetration of two planar rigid bodies, combined with the Coulomb friction model result in the definition of four different contact modes for each contact [18]: separating $(S)$, sliding left $(L)$ or right $(R)$ or fixed $(F)$. Each contact point hence has an associated contact mode

$$
c_{i} \in \mathcal{M}:=\{S, L, R, F\}
$$

Depending on the mode and velocity of a particular contact, a set of constraints on accelerations and reaction forces of the corresponding contact point must be satisfied. These constraints are most easily written in local contact

\footnotetext{
${ }^{2}$ Note that we use the subscript $e$ to distinguish definitions related
} to the example system from their generic counterparts. 
TABLE I

Normal Force and Acceleration Constraints

\begin{tabular}{|c|c|c|c|}
\hline Mode & Velocity & Acceleration & $f_{\mathbf{n}_{i}}$ \\
\hline \hline $\mathrm{S}$ & $\dot{x}_{\mathbf{n}_{i}}>0$ & - & $f_{\mathbf{n}_{i}}=0$ \\
\hline $\mathrm{S}$ & $\dot{x}_{\mathbf{n}_{i}}=0$ & $\ddot{x}_{\mathbf{n}_{i}} \geq 0$ & $f_{\mathbf{n}_{i}}=0$ \\
\hline F,L,R & $\dot{x}_{\mathbf{n}_{i}}=0$ & $\ddot{x}_{\mathbf{n}_{i}}=0$ & $f_{\mathbf{n}_{i}} \geq 0$ \\
\hline
\end{tabular}

TABLE II

Tangential Force and AcCeleration Constraints

\begin{tabular}{|c|c|c|c|}
\hline Mode & Velocity & Acceleration & $f_{\mathbf{t}_{i}}$ \\
\hline \hline $\mathrm{R}$ & $\dot{x}_{\mathbf{t}_{i}}>0$ & - & $f_{\mathbf{t}_{i}}=-\mu f_{\mathbf{n}_{i}}$ \\
\hline $\mathrm{L}$ & $\dot{x}_{\mathbf{t}_{i}}<0$ & - & $f_{\mathbf{t}_{i}}=\mu f_{\mathbf{n}_{i}}$ \\
\hline $\mathrm{R}$ & $\dot{x}_{\mathbf{t}_{i}}=0$ & $\ddot{x}_{\mathbf{t}_{i}} \geq 0$ & $f_{\mathbf{t}_{i}}=-\mu f_{\mathbf{n}_{i}}$ \\
\hline $\mathrm{L}$ & $\dot{x}_{\mathbf{t}_{i}}=0$ & $\ddot{x}_{\mathbf{t}_{i}} \leq 0$ & $f_{\mathbf{t}_{i}}=\mu f_{\mathbf{n}_{i}}$ \\
\hline $\mathrm{F}$ & $\dot{x}_{\mathbf{t}_{i}}=0$ & $\ddot{x}_{\mathbf{t}_{i}}=0$ & $\left\|f_{\mathbf{t}_{i}}\right\| \leq \mu f_{\mathbf{n}_{i}}$ \\
\hline
\end{tabular}

coordinates and are summarized in Tables I and II. If the contact point is separating (S), either due to velocity or acceleration, then no reaction force is allowed, otherwise a compressive normal force is possible. Given a contact point which is not separating, we also consider its tangential motion. If the contact point is sliding left $(\mathrm{L})$ or right $(\mathrm{R})$ with respect to its local coordinate frame, then the tangential force must be at a maximum, otherwise the contact point is fixed $(\mathrm{F})$ and the tangential force can be anywhere in the friction cone. Throughout the rest of the paper, we denote the overall contact mode of the system as a vector of individual modes for each contact

$$
\mathbf{c}:=\left[c_{1}, \ldots, c_{k}\right] \in \mathcal{M}^{k} .
$$

In subsequent sections, we will also find it useful to encode the constraints of Tables I and II in matrix form. The following definitions are presented for a given overall contact mode for the system. Without loss of generality, we order and partition the contact Jacobian $\mathbf{J}$ as

$$
\mathbf{J}=\left[\begin{array}{c}
\mathbf{J}_{F} \\
\mathbf{J}_{S} \\
\mathbf{J}_{L} \\
\mathbf{J}_{R}
\end{array}\right]
$$

according to the individual contacts that are in specific modes. For equality constraints on system accelerations, we can use the appropriate rows of (4) to yield

$$
\left[\begin{array}{c}
\mathbf{J}_{F} \\
\mathbf{J}_{L_{n}} \\
\mathbf{J}_{R_{n}}
\end{array}\right] \ddot{\mathbf{q}}+\left[\begin{array}{c}
\dot{\mathbf{J}}_{F} \\
\dot{\mathbf{J}}_{L_{n}} \\
\dot{\mathbf{J}}_{R_{n}}
\end{array}\right] \dot{\mathbf{q}}=\left[\begin{array}{l}
0 \\
0 \\
0
\end{array}\right] .
$$

Similarly, for equality constraints on the contact reaction forces, we have

$$
\left[\begin{array}{cccccc}
\mathbf{0} & \mathbf{I}_{\mathbf{2}} & \mathbf{0} & \mathbf{0} & \mathbf{0} & \mathbf{0} \\
\mathbf{0} & \mathbf{0} & -\mu \mathbf{I}_{\mathbf{L}} & \mathbf{I}_{\mathbf{L}} & \mathbf{0} & \mathbf{0} \\
\mathbf{0} & \mathbf{0} & \mathbf{0} & \mathbf{0} & \mu \mathbf{I}_{\mathbf{R}} & \mathbf{I}_{\mathbf{R}}
\end{array}\right]\left[\begin{array}{c}
\mathbf{f}_{F} \\
\mathbf{f}_{S} \\
\mathbf{f}_{L_{n}} \\
\mathbf{f}_{L_{t}} \\
\mathbf{f}_{R_{n}} \\
\mathbf{f}_{R_{t}}
\end{array}\right]=0
$$

where $\mathbf{I}_{\mathbf{L}}$ is the identity matrix of size equal to the number of left sliding contact points, for example. The remaining inequality constraints from Tables I and II take the form

$$
\left[\begin{array}{c}
\mathbf{J}_{S_{n}} \\
-\mathbf{J}_{L_{t}} \\
\mathbf{J}_{R_{t}}
\end{array}\right] \ddot{\mathbf{q}}+\left[\begin{array}{c}
\dot{\mathbf{J}}_{S_{n}} \\
-\dot{\mathbf{J}}_{L_{t}} \\
\dot{\mathbf{J}}_{R_{t}}
\end{array}\right] \dot{\mathbf{q}} \geq 0
$$

for accelerations and

$$
\left[\begin{array}{ccccccc}
\mu \mathbf{I}_{\mathbf{F}} & -\mathbf{I}_{\mathbf{F}} & \mathbf{0} & \mathbf{0} & \mathbf{0} & \mathbf{0} & \mathbf{0} \\
\mu \mathbf{I}_{\mathbf{F}} & \mathbf{I}_{\mathbf{F}} & \mathbf{0} & \mathbf{0} & \mathbf{0} & \mathbf{0} & \mathbf{0} \\
\mathbf{0} & \mathbf{0} & \mathbf{0} & \mathbf{I}_{\mathbf{L}} & \mathbf{0} & \mathbf{0} & \mathbf{0} \\
\mathbf{0} & \mathbf{0} & \mathbf{0} & \mathbf{0} & \mathbf{0} & \mathbf{I}_{\mathbf{R}} & \mathbf{0}
\end{array}\right]\left[\begin{array}{c}
\mathbf{f}_{F_{n}} \\
\mathbf{f}_{F_{t}} \\
\mathbf{f}_{S} \\
\mathbf{f}_{L_{n}} \\
\mathbf{f}_{L_{t}} \\
\mathbf{f}_{R_{n}} \\
\mathbf{f}_{R_{t}}
\end{array}\right] \geq 0
$$

for contact reaction forces. Acceleration inequalities of (9) are only applicable to separating contact points with zero normal velocity and sliding contact points with zero tangential velocity as indicated in the Tables I and II.

In this paper, we also consider the possibility of imposing additional model specific linear constraints, which we divide into two categories: physical constraints and task constraints.

Physical constraints are constraints which are physically required to hold for all dynamic solutions. For example, in the flipping application presented in Section IV, we assume 
all legs to be massless. This induces an algebraic constraint between the applied hip control torque and contact reaction forces associated with the corresponding leg. More formally, physical constraints are assumed to be specified through a set of equalities of the form

$$
\begin{aligned}
& \mathbf{A}_{p}^{\ddot{\mathbf{q}}} \ddot{\mathbf{q}}=\mathbf{B}_{p}^{\ddot{\mathbf{q}}} \tau+\mathbf{b}_{p}^{\ddot{\mathbf{q}}} \\
& \mathbf{A}_{p}^{\mathbf{f}} \mathbf{f}=\mathbf{B}_{p}^{\mathbf{f}} \tau+\mathbf{b}_{p}^{\mathbf{f}},
\end{aligned}
$$

as well as a set of inequalities of the form

$$
\begin{aligned}
& \mathbf{N}_{p}^{\ddot{\mathbf{q}}} \ddot{\mathbf{q}} \geq \mathbf{E}_{p}^{\ddot{\mathbf{q}}} \tau+\mathbf{e}_{p}^{\ddot{\mathbf{q}}} \\
& \mathbf{N}_{p}^{\mathbf{f}} \mathbf{f} \geq \mathbf{E}_{p}^{\mathbf{f}} \tau+\mathbf{e}_{p}^{\mathbf{f}} .
\end{aligned}
$$

In contrast, task constraints are user imposed conditions that are not physically necessary, but rather application specific, desired properties of dynamic solutions. In our flipping example, we use this type of constraint to impose a minimum threshold on the normal component of the reaction force for the body-ground contact, ensuring maintained body contact throughout flipping. Task constraints are external to the model and deviate from our original goal of finding the overall dynamic response of the form (2). They restrict the solutions in task specific ways and require additional care in the characterization of contact mode ambiguities as well as solutions to the dynamics. Even though we are able to incorporate such constraints very naturally in our modeling tool, the reader should keep in mind that they result in unnatural restrictions on the identified solution domains for the dynamic response. A more detailed account of the impact of task constraints on mode ambiguities is presented in Section III-G.1. Task constraints are assumed to be specified through a set of equalities and inequalities of the form

$$
\begin{aligned}
\mathbf{A}_{t}^{\ddot{\mathbf{q}}} \ddot{\mathbf{q}} & =\mathbf{B}_{t}^{\ddot{\mathbf{q}}} \tau+\mathbf{b}_{t}^{\ddot{\mathbf{q}}} \\
\mathbf{A}_{t}^{\mathbf{f}} \mathbf{f} & =\mathbf{B}_{t}^{\mathbf{f}} \tau+\mathbf{b}_{t}^{\mathbf{f}},
\end{aligned}
$$

and

$$
\begin{aligned}
\mathbf{N}_{t}^{\ddot{\mathbf{q}}} \ddot{\mathbf{q}} & \geq \mathbf{E}_{t}^{\ddot{\mathbf{q}}} \tau+\mathbf{e}_{t}^{\ddot{\mathbf{q}}} \\
\mathbf{N}_{t}^{\mathbf{f}} \mathbf{f} & \geq \mathbf{E}_{t}^{\mathbf{f}} \tau+\mathbf{e}_{t}^{\mathbf{f}} .
\end{aligned}
$$

For clarity of presentation, we will find it convenient to merge all the equality constraints in $(7),(8),(11),(12),(15)$ and (16) into two systems of equations for accelerations and reaction forces

$$
\begin{aligned}
& \mathbf{A}^{\ddot{\mathbf{q}}} \ddot{\mathbf{q}}=\mathbf{B}^{\ddot{\mathbf{q}}} \tau+\mathbf{b}^{\ddot{\mathbf{q}}} \\
& \mathbf{A}^{\mathbf{f}} \mathbf{f}=\mathbf{B}^{\mathbf{f}} \tau+\mathbf{b}^{\mathbf{f}} \text {. }
\end{aligned}
$$

Similarly, we merge all inequality constraints in (9), (10), (13), (14), (17) and (18) into two systems of inequalities

$$
\begin{aligned}
& \mathbf{N}^{\ddot{\mathbf{q}}} \ddot{\mathbf{q}} \geq \mathbf{E}^{\ddot{\mathbf{q}}} \tau+\mathbf{e}^{\ddot{\mathbf{q}}} \\
& \mathbf{N}^{\mathbf{f}} \mathbf{f} \geq \mathbf{E}^{\mathbf{f}} \tau+\mathbf{e}^{\mathbf{f}} .
\end{aligned}
$$

Returning to the two-link example of Figure 1, we refer to Tables I and II to derive the required contact constraints. Suppose, for instance, that the system has no initial velocity $\left(\dot{\mathbf{q}}_{\mathbf{e}}=\mathbf{0}\right)$ and that all contacts are fixed $(\mathrm{F})$. As a result, we have acceleration constraints $\ddot{x}_{\mathbf{n}_{i}}=\ddot{x}_{\mathbf{t}_{i}}=0$ and force constraints $f_{\mathbf{n}_{i}} \geq 0,\left\|f_{\mathbf{t}_{i}}\right\| \leq \mu f_{\mathbf{n}_{i}}$ for each contact point. In the absence of any physical or task constraints, matrices for the equality constraints of (19) take the form

$$
\mathbf{A}_{\mathbf{e}}^{\ddot{\mathrm{q}}}=\mathbf{J}_{\mathbf{e}}, \quad \mathbf{B}_{\mathbf{e}}^{\ddot{\mathrm{q}}}=\mathbf{0}_{6 \times 1}, \quad \mathbf{b}_{\mathbf{e}}^{\ddot{\mathrm{q}}}=\mathbf{0}_{6 \times 1},
$$

and the matrices for the inequality constraints of (22) yield

$$
\mathbf{N}_{\mathbf{e}}^{\mathbf{f}}=\left[\begin{array}{cc}
\mu \mathbf{I}_{3} & -\mathbf{I}_{3} \\
\mu \mathbf{I}_{3} & \mathbf{I}_{3}
\end{array}\right], \quad \mathbf{E}_{\mathbf{e}}^{\mathbf{f}}=\mathbf{0}_{\mathbf{6 \times 1}}, \quad \mathbf{e}_{\mathbf{e}}^{\mathbf{f}}=\mathbf{0}_{\mathbf{6} \times \mathbf{1}}
$$

In contrast, the systems expressed in (20) and (21) are empty, since there are no associated constraints. All kinematically feasible combinations of contact modes have similar derivations for their associated constraint equations.

Note that in this paper we do not consider impact models, precluding us from computing solutions to the system whenever $\dot{x}_{\mathbf{n}_{i}}<0$ or $\ddot{x}_{\mathbf{n}_{i}}<0$. Among other ramifications, Painlevé type problems [9], [18], [31] where sliding friction would result in rigid-body penetration will thus have no solution, and ambiguities due to impacts are ignored. This limits the applicability of our methods to situations where the consideration of such impacts is not important. On the other hand, the simplifications that result from our assumption are significant and useful if impacts are not critical for a particular application. 


\section{Form of Solutions and Outline of the Algorithm}

Given a particular contact mode vector, $\mathbf{c}:=\left[c_{1}, \ldots, c_{k}\right] \in$ $\mathcal{M}^{k}$, and control inputs $\tau$, we denote by $\mathbf{g}_{\mathbf{c}}(\tau, \mathbf{w})$, the instantaneous dynamic response of the system within a contact mode c, defined as the solution to (5), subject to the constraints (19), (20), (21) and (22). In order to construct the final form of the dynamic response in (2), we partition its domain into a discrete number of cells, each corresponding to a subset of the global domain $\mathcal{D}_{\mathbf{c}} \subseteq \mathbb{R}^{t} \times \mathbb{R}^{w}$, wherein a particular choice of contact mode can be realized. The general for of the dynamic response hence takes the form

$$
\mathbf{g}(\tau, \mathbf{w}):= \begin{cases}\mathbf{g}_{\mathbf{c}_{1}}(\tau, \mathbf{w}) & \text { if }(\tau, \mathbf{w}) \in \mathcal{D}_{\mathbf{c}_{1}} \\ \cdots & \\ \mathbf{g}_{\mathbf{c}_{\left(4^{k}\right)}}(\tau, \mathbf{w}) & \text { if }(\tau, \mathbf{w}) \in \mathcal{D}_{\mathbf{c}_{\left(4^{k}\right)}}\end{cases}
$$

where we have dropped the dependence on $(\mathbf{q}, \dot{\mathbf{q}})$ for notational convenience. Note that each component of this dynamic response is linear in control inputs and ambiguity variables, resulting in a function which is piecewise linear over its domain.

The primary contribution of this paper is the computation of mode specific response functions $\mathbf{g}_{\mathbf{c}}(\tau, \mathbf{w})$ together with their domains of validity $\mathcal{D}_{\mathbf{c}}$. We also compute projections of individual domains on to the torque axes to obtain a concise representation in the space of control inputs. Note that due to the presence of ambiguities and inconsistencies of solutions, neither the sets $\mathcal{D}_{\mathbf{c}}$ nor their projections are guaranteed to be disjoint or even cover the entire input torque space. Finally, in the presence of task constraints, we will find it useful to compute the dynamic response both with and without task constraints, allowing for a full characterization of ambiguities as described in Section III-G.

Each of the mode specific dynamic response functions in (23) as well as the associated equality and inequality constraints described in Section III-B are linear. As a consequence, our algorithm is based on three linear operations:

1. Solving a linear system (SVD)

2. Computing Support Hyperplanes (Solving a LP)

3. Polytope Projection (Fourier-Motzkin)

In particular, we start by solving the equality constraints, allowing us to compute $\mathbf{g}_{\mathbf{c}}(\tau, \mathbf{w})$. The remaining inequality constraints describe the domain of validity for this function and can be reduced to a simple polytope in the space of control inputs and ambiguity variables through linear programming. Finally, we project the resulting polytope onto the control input space yielding the set of feasible control inputs for each mode.

The decomposition of the entire problem into a set of linear subproblems also allows us to construct effective algorithms for task specific goals such as optimization over the solution set. We illustrate a specific example of such an application domain in Section IV for the dynamic selfrighting of a hexapod robot. However, it is important to note that such decompositions can induce potentially prohibitive computational costs, typical of enumerative methods on problems with a large number of contacts.

\section{Computing the Mode Specific Dynamic Response}

Given a contact mode, three sets of linear equality constraints must be satisfied by the unknown forces and accelerations of the system. The first set comes directly from the Lagrangian formulation in (5). The remaining two, (19) and (20), are constraints on system accelerations and forces, respectively, and originate from the contact, physical, and task constraints of Section III-B.

Our approach is to simultaneously solve these linear equations to calculate $\mathbf{g}_{\mathbf{c}}(\tau, \mathbf{w})$. In doing so, we are able to parametrically characterize force and acceleration ambiguities associated with the chosen contact mode and produce a closed form solution for the dynamic response as a function of the control inputs and ambiguity variables.

Before we proceed, we collect all linear equality constraints on contact forces and system accelerations as well as the dynamic constraint equation in a single large linear system $^{3}$

$$
\left[\begin{array}{cc}
\mathbf{A}^{\ddot{\mathbf{q}}} & \mathbf{0} \\
\mathbf{0} & \mathbf{A}^{\mathbf{f}} \\
\mathbf{M}(\mathbf{q}) & -\mathbf{J}^{T}
\end{array}\right]\left[\begin{array}{c}
\ddot{\mathbf{q}} \\
\mathbf{f}
\end{array}\right]=\left[\begin{array}{c}
\mathbf{B}^{\ddot{q}} \\
\mathbf{B}^{\mathbf{f}} \\
\mathbf{I}
\end{array}\right] \tau+\left[\begin{array}{c}
\mathbf{b}^{\ddot{\mathbf{q}}} \\
\mathbf{b}^{\mathbf{f}} \\
\mathbf{b}^{d y n}
\end{array}\right]
$$

${ }^{3}$ Note that for computational efficiency, it would be beneficial to exploit the structure in the three different groups of constraints by first solving force constraints, then solving acceleration constraints, finally followed by substitution into and solution of the dynamic constraint of (5). 
where $\mathbf{b}^{d y n}:=-\mathbf{g}(\mathbf{q})-\mathbf{C}(\mathbf{q}, \dot{\mathbf{q}}) \dot{\mathbf{q}}$. In subsequent derivations, this equation will be used in its simpler notational form

$$
\mathbf{A}\left[\begin{array}{c}
\ddot{\mathbf{q}} \\
\mathbf{f}
\end{array}\right]=\mathbf{B} \tau+\mathbf{b}
$$

Different possible rank conditions of the matrix $\mathbf{A}$ result in different forms for the solutions to this linear system. In particular, we identify four cases as all possible combinations of full column rank (f.c.r) and full row rank (f.r.r) properties of $\mathbf{A}$.

1. A is both f.r.r. and f.c.r.

In this case, $\mathbf{A}$ is invertible and there is a globally defined unique solution for $(\ddot{\mathbf{q}}, \mathbf{f})$ as a function of torque, taking the form

$$
\left[\begin{array}{c}
\ddot{\mathbf{q}} \\
\mathbf{f}
\end{array}\right]=\mathbf{A}^{-1} \mathbf{B} \tau+\mathbf{A}^{-1} \mathbf{b} .
$$

\section{A is f.r.r. but not f.c.r.}

In this case, A is not invertible, and has some null space. As a consequence, even though the response function is defined for all control inputs $\tau$, multiple solutions exist and they take the form

$$
\left[\begin{array}{c}
\ddot{\mathbf{q}} \\
\mathbf{f}
\end{array}\right]=\mathbf{A}^{\dagger} \mathbf{B} \tau+\mathbf{A}^{\dagger} \mathbf{b}+\mathbf{N}(\mathbf{A}) \mathbf{w},
$$

where $\mathbf{A}^{\dagger}$ and $\mathbf{N}(\mathbf{A})$ denote the pseudo-inverse and a basis for the nullspace of $\mathbf{A}$, respectively, and $\mathbf{w}$ provides a parametrization of solution ambiguities.

3. A is f.c.r. but not is f.r.r.

In this case, $\mathbf{A}$ is not invertible, but does not have a nullspace. Consequently, solutions are unique when they exist. On the other hand, since $\mathbf{A}$ is not full row rank, there may be control inputs for which no solutions can be found. This happens whenever the affine subspace spanned by the right hand side of (25) is not contained in the column space of $\mathbf{A}$. In such cases, we must explicitly solve for the subspace of control inputs for which the system admits a solution. For the time being, let us denote this subspace as $\tau=\mathbf{A}^{p} \tau_{p}+\mathbf{b}^{p}$ where $\tau_{p}$ is a parameterization of the admissible torque subspace. The dynamic response function then takes the form

$$
\begin{gathered}
{\left[\begin{array}{c}
\ddot{\mathbf{q}} \\
\mathbf{f}
\end{array}\right]=\mathbf{A}^{\dagger} \mathbf{B} \tau+\mathbf{A}^{\dagger} \mathbf{b}} \\
\text { s.t. } \tau=\mathbf{A}^{p} \tau_{p}+\mathbf{b}^{p} .
\end{gathered}
$$

Later in this section, we will provide an algorithm to compute $\mathbf{A}^{p}$ and $\mathbf{b}^{p}$.

4. $\mathbf{A}$ is neither f.r.r. nor f.c.r.

In this final case, not only is $\mathbf{A}$ not invertible, but it also has a nullspace leading to multiple solutions and potential non-existence of solutions due to its deficient row rank. The solution for $(\ddot{\mathbf{q}}, \mathbf{f})$ takes the form

$$
\begin{array}{r}
{\left[\begin{array}{c}
\ddot{\mathbf{q}} \\
\mathbf{f}
\end{array}\right]=\mathbf{A}^{\dagger} \mathbf{B} \tau+\mathbf{A}^{\dagger} \mathbf{b}+\mathbf{N}(\mathbf{A}) \mathbf{w}} \\
\text { s.t. } \tau=\mathbf{A}^{p} \tau_{p}+\mathbf{b}^{p} .
\end{array}
$$

For the two cases where the matrix $\mathbf{A}$ is row rank deficient, we need to solve for the affine subspace of control inputs that admit a solution for the dynamic response. This is easily accomplished by rearranging the linear system (25) and considering the control torques as a variable

$$
\left[\begin{array}{ll}
\mathbf{A} & \mathbf{B}
\end{array}\right]\left[\begin{array}{c}
\ddot{\mathbf{q}} \\
\mathbf{f} \\
\tau
\end{array}\right]=\mathbf{b}
$$

Using the notation \{\}$_{\tau}$ to refer to the last rows of coefficient matrices in the solution, we have

$$
\tau=\left\{\left[\begin{array}{ll}
\mathbf{A} & \mathbf{B}
\end{array}\right]^{\dagger}\right\}_{\tau} \mathbf{b}+\left\{\mathbf{N}\left(\left[\begin{array}{ll}
\mathbf{A} & \mathbf{B}
\end{array}\right]\right)\right\}_{\tau} \tau_{p}
$$

concisely parameterizing admissible control inputs for which the dynamics admit an instantaneous solution. Note that the potential reduction in the actuation freedom through the smaller parameterization $\tau_{p}$ must be taken into account in computing the domain of $\mathbf{g}_{\mathbf{c}}(\tau, \mathbf{w})$ in the next section. This can either be done by rewriting the domain on the reduced set of coordinates or simply keeping track of the reduced torque subspace.

To illustrate the algorithm, consider once again the example two-link system with all three contact points in fixed mode and $\tau_{\gamma}<0$. Collecting previously defined quantities 
yields the matrices in (25) as

$$
\begin{aligned}
\mathbf{A}_{\mathbf{e}} & :=\left[\begin{array}{cc}
\mathbf{J}_{\mathbf{e}} & \mathbf{0} \\
\mathbf{M}_{\mathbf{e}} & -\mathbf{J}_{\mathbf{e}}^{T}
\end{array}\right], \quad \mathbf{B}_{\mathbf{e}}:=\left[\begin{array}{ll}
\mathbf{0}_{\mathbf{1 \times 6} 6} & 1
\end{array}\right]^{T} \\
\mathbf{b}_{\mathbf{e}} & :=\left[\begin{array}{ll}
\mathbf{0}_{\mathbf{1 \times 6} \mathbf{6}} & \mathbf{b}_{e}^{d y n}
\end{array}\right]^{T} .
\end{aligned}
$$

In the configuration shown in Figure 1, the matrix $\mathbf{A}_{\mathbf{e}} \in \mathbb{R}^{10 \times 10}$ is only rank 8 , due to the rank deficiency of $\mathbf{J}_{\mathbf{e}}^{T}$ and hence falls into case 4 above. The multiplicity of solutions within the mode is characterized by the nullspace component of (29), parameterized in this case by two ambiguity variables $\mathbf{w}_{\mathbf{e}}:=\left(w_{1}, w_{2}\right)$. Intuitively, the compressive forces along either of the links cannot be determined with a rigid model, yielding a two-dimensional space of possible reaction forces for any given control input $\tau_{\gamma}$. Note that, in this example, the affine subspace spanned by $\mathbf{B}_{\mathbf{e}} \tau_{\gamma}+\mathbf{b}_{\mathbf{e}}$ lies in the column space of $\mathbf{A}_{\mathbf{e}}$, and hence solutions exist for the most general set of possible control inputs $\tau_{\gamma}$.

\section{E. Computing the Domain of the Dynamic Response}

As described in the previous section, the solutions to the Lagrangian dynamics under equality constraints associated with a particular contact mode yields the functional form of the local dynamic response function $\mathbf{g}_{\mathbf{c}}(\tau, \mathbf{w})$, mapping control inputs and ambiguity variables to system accelerations and reaction forces. It still remains, however, to incorporate all inequality constraints introduced in Section III-B. Our interpretation of these remaining constraints is a restriction on the functional domain of $\mathbf{g}_{\mathbf{c}}(\tau, \mathbf{w})$. All equality and inequality constraints being linear, this domain $\mathcal{D}_{\mathbf{c}}$, can be effectively represented as a convex polytope in the space of control inputs and ambiguity variables.

Similar to our treatment of equality constraints, we group both reaction force and acceleration inequalities of (22) and (21) into a single system

$$
\left[\begin{array}{ccc}
\mathbf{N}^{\ddot{\mathbf{q}}} & \mathbf{0} & \mathbf{E}^{\ddot{\mathrm{q}}} \\
\mathbf{0} & \mathbf{N}^{\mathrm{f}} & \mathbf{E}^{\mathbf{f}}
\end{array}\right]\left[\begin{array}{c}
\ddot{\mathbf{q}} \\
\mathbf{f} \\
\tau
\end{array}\right] \geq\left[\begin{array}{c}
\mathbf{e}^{\ddot{\mathbf{q}}} \\
\mathbf{e}^{\mathbf{f}}
\end{array}\right] .
$$

The appropriate choice out of the four possible closed form solutions in (26), (27), (28), or (29) from the previous section can be directly substituted in this system of inequalities, effectively eliminating the generalized system accelerations $\ddot{\mathbf{q}}$ and contact forces $\mathbf{f}$. We are then left with a set of linear inequality constraints on the control inputs $\tau$ and ambiguity variables $\mathbf{w}$, taking the form

$$
\mathbf{N}\left[\begin{array}{c}
\tau \\
\mathbf{w}
\end{array}\right] \geq \mathbf{e}
$$

For instance, suppose that the solution to the dynamic response falls into the fourth case, shown in (29). In that case, simple substitution into (32) yields

$$
\left[\begin{array}{ll}
\mathbf{N}_{1} \mathbf{A}^{\dagger} \mathbf{B}+\mathbf{E}_{1} & \mathbf{N}_{1} \mathbf{N}(\mathbf{A})
\end{array}\right]\left[\begin{array}{c}
\tau \\
\mathbf{w}
\end{array}\right] \geq \mathbf{e}_{1}-\mathbf{N}_{1} \mathbf{A}^{\dagger} \mathbf{b},
$$

which conforms to the generic form of (33) with the definitions

$$
\mathbf{N}_{1}:=\left[\begin{array}{cc}
\mathbf{N}^{\ddot{\mathbf{q}}} & \mathbf{0} \\
\mathbf{0} & \mathbf{N}^{\mathbf{f}}
\end{array}\right], \quad \mathbf{E}_{1}:=\left[\begin{array}{l}
\mathbf{E}^{\ddot{\mathbf{q}}} \\
\mathbf{E}^{\mathbf{f}}
\end{array}\right], \quad \mathbf{e}_{1}:=\left[\begin{array}{c}
\mathbf{e}^{\ddot{\mathbf{q}}} \\
\mathbf{e}^{\mathbf{f}}
\end{array}\right] .
$$

By definition, all control torques and ambiguity variables satisfying this system of inequalities result in reaction forces and system accelerations that are guaranteed to be consistent with the assumed contact mode. We hence define the domain of the dynamic response $\mathcal{D}_{\mathbf{c}}$ to be precisely the set of solutions to the system in (33). Furthermore, it turns out that the most effective and useful representation for this domain is a reduced set of inequalities which only retains supporting constraints that intersect the boundary of the solution set. This reduction can easily be accomplished by solving a linear program for each individual inequality, yielding a concise representation for the domain.

Consider for instance, the $i^{\text {th }}$ row of (33),

$$
\mathbf{n}^{\mathbf{i}}\left[\begin{array}{c}
\tau \\
\mathbf{w}
\end{array}\right] \geq \mathbf{e}_{\mathbf{i}}
$$

In order to determine whether this is a supporting hyper- 
plane, we solve the linear program

$$
\begin{gathered}
\min x \\
\text { s.t. : }\left[\begin{array}{ll}
\mathbf{N} & 1
\end{array}\right]\left[\begin{array}{c}
\tau \\
\mathbf{w} \\
x
\end{array}\right] \geq \mathbf{e} \\
\text { and }\left[\begin{array}{ll}
\mathbf{n}^{\mathbf{i}} & 0
\end{array}\right]\left[\begin{array}{c}
\tau \\
\mathbf{w} \\
x
\end{array}\right]=\mathbf{e}_{\mathbf{i}} .
\end{gathered}
$$

The constraint under consideration yields a supporting half-plane if and only if the optimal solution $x$ to this linear program is zero [34]. Performing this test on all inequality constraints, we can eliminate all non-supporting hyperplanes in the representation of $\mathcal{D}_{\mathbf{c}}$. Not surprisingly, if there are no supporting constraints, then $\mathcal{D}_{\mathbf{c}}=\emptyset$.

Our main motivation is the design of controllers for mechanical systems under frictional contact. Consequently, once we have computed a simplified representation of $\mathcal{D}_{\mathbf{c}}$, we then compute its projection, $\mathcal{T}_{\mathbf{c}}$, onto the $\tau$ axes, shown in Figure 2. This projection yields the set of control torques that are consistent with (i.e. could result in) a given contact mode and may be computed by using a polytope projection technique such as Fourier-Motzkin [15]. The process is similar to Gaussian elimination but deals with linear inequalities rather than equalities. Briefly, elimination of a variable with the Fourier-Motzkin technique involves adding together all permutations of inequalities with coefficients of opposite sign on the variable to be eliminated. The inequalities are scaled by a positive scalar so that these opposite sign coefficients cancel, thus eliminating the particular variable. The projection process often produces redundant constraints and an additional reduction through linear programming may be required to eliminate redundant non-supporting hyperplanes.

Consider once again, the two-link example of Figure 1 with all three contact points fixed. In this case, the domain for the dynamic response, $\mathcal{D}_{\mathbf{c}}$, is represented as a threedimensional polytope in the space of the elbow torque and the two ambiguity variables, taking the form

$$
\mathbf{N}_{\mathbf{e}}\left[\begin{array}{lll}
\tau_{\gamma} & w_{1} & w_{2}
\end{array}\right]^{T} \geq 0
$$

$\mathbf{N}_{\mathrm{e}}$ is obtained by straightforward substitution into (32),

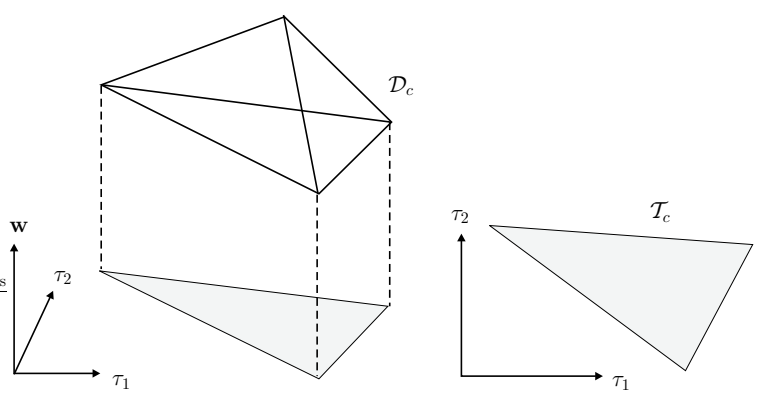

Fig. 2. The projection of $\mathcal{D}_{\mathbf{c}}$ by Fourier-Motzkin eliminates ambiguity variables. The resultant set $\mathcal{T}_{\mathbf{c}}$ characterizes the domain with respect to the control torques.

\begin{tabular}{|c|c|c|}
\hline Normal Velocity & Tangential Velocity & Modes \\
\hline \hline$\dot{x}_{\mathbf{n}_{i}}>0$ & --- & $\mathrm{S}$ \\
\hline$\dot{x}_{\mathbf{n}_{i}}=0$ & $\dot{x}_{\mathbf{t}_{i}}>0$ & $\mathrm{~S}, \mathrm{R}$ \\
\hline$\dot{x}_{\mathbf{n}_{i}}=0$ & $\dot{x}_{\mathbf{t}_{i}}<0$ & $\mathrm{~S}, \mathrm{~L}$ \\
\hline$\dot{x}_{\mathbf{n}_{i}}=0$ & $\dot{x}_{\mathbf{t}_{i}}=0$ & $\mathrm{~F}, \mathrm{~S}, \mathrm{~L}, \mathrm{R}$ \\
\hline
\end{tabular}

TABLE III

KINEMATICALLY FEASIBLE CONTACT MODES BASED ON CONTACT POINT VELOCITY

omitted here for space considerations. For a fixed control torque, this polytope encodes the range of possible reaction forces by limiting the values of $w_{1}$ and $w_{2}$. For small coefficients of friction $\mu$, this range will be small, and for large coefficients this range becomes unbounded as the link falls "inside" the friction cone. Projection of this polytope onto the torque input space yields a one-dimensional range of controls for which all contact points are sticking (up to ambiguities between different mode, discussed in the next section).

\section{F. Enumeration of Modes and Kinematic Feasibility}

The most general form of the dynamic response function (23) requires the consideration of all $\left|\mathcal{M}^{k}\right|=4^{k}$ possible contact modes of the system. Using the algorithms presented in Sections III-D and III-E, this would involve computing functions $\mathbf{g}_{\mathbf{c}}(\tau, \mathbf{w})$ and their domains $\mathcal{D}_{\mathbf{c}}$ for each contact mode.

Fortunately, we can significantly decrease the number of contact modes for which these quantities have to be 
computed by taking into account kinematic components of the constraints in Section III-B. For any given contact, its normal and tangential velocities computed through the jacobian map of (3) helps us determine which modes are kinematically feasible. Table III-F summarizes all possible velocity based eliminations ${ }^{4}$.

In addition to local velocity eliminations for each contact, it is possible to rule out contact modes based on constraints on system kinematics across all contacts. In particular, the acceleration constraints in Tables I and II are constrained to be consistent across all contact points. Mode combinations that violate this constraint can be identified through a linear programming problem and are most commonly observed when the number of contacts is large with respect to the internal degrees of freedom in the system $^{5}$.

Local and global elimination of infeasible modes in this manner results in a smaller set of contact modes to be considered in our algorithm. We define the resulting collection of contact modes to be the set of kinematically feasible contact modes, denoted by $\mathcal{M}_{p} \subseteq \mathcal{M}^{k}$. Note that this feasible set depends only on the current system configuration and velocities $\mathbf{q}$ and $\dot{\mathbf{q}}$.

As an illustrative example, consider the two-link system of Figure 1 with the top right contact point sliding down (L) and the remaining two contact points fixed. This contact mode requires that $\ddot{x}_{\mathbf{n}_{1}}=0, \ddot{x}_{\mathbf{t}_{1}}<0$ and $\ddot{x}_{\mathbf{n}_{2}}=0, \ddot{x}_{\mathbf{t}_{2}}=0$. Converting these constraints to the generalized accelerations $\ddot{\mathbf{q}}$ though the jacobian and testing for solutions with a linear program reveals that this contact mode is kinematically infeasible. Intuitively, once a point on a single link is fixed, the remainder of the link can only move by pivoting about this point and cannot slide in an arbitrary direction.

\footnotetext{
${ }^{4}$ In practice, since exact comparison of velocity measurements with zero is not possible, additional filtering may be necessary to determine which contact modes ought to be considered as feasible.

${ }^{5}$ In applying this elimination, acceleration inequalities associated with modes $\mathrm{S}, \mathrm{L}$, and $\mathrm{R}$ are treated as strict inequalities, since the zero acceleration case is captured by the fixed mode $\mathrm{F}$.
}

\section{G. Characterizing Solution Ambiguities}

A typical problem associated with Coulomb frictional models is potential ambiguities in computing solutions. In Section III-D, we have been able to parameterize potential acceleration and force ambiguities within a particular contact mode by introducing a vector of ambiguity variables, w. An additional possible source of ambiguities is in determining the contact mode realized by a particular choice of control inputs. In this section, we carefully characterize each of these two types of ambiguities, illustrated in Figure 3 , and present methods useful in the design of controllers that can avoid associated problems.
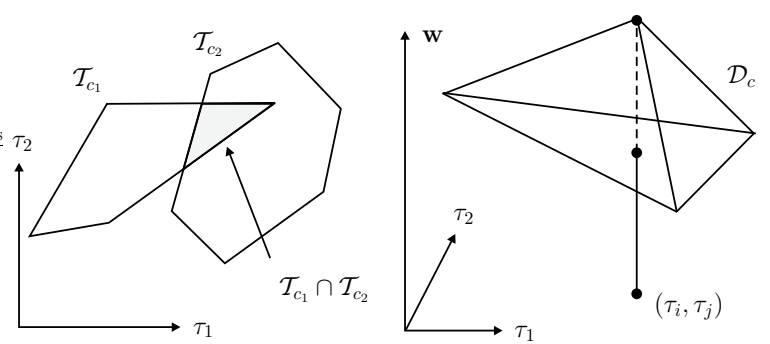

Fig. 3. The two types of solution ambiguities. The figure on the left illustrates an ambiguity between two contact modes, manifested as an intersection of the control input domains $\mathcal{T}_{\mathbf{c}}$. The figure on the right illustrates ambiguities within a contact mode, characterized by the feasible range in the variable $\mathbf{w}$ for a given control input.

Our distinction between solution ambiguities within a particular mode and contact mode ambiguities closely parallels our decomposition of the problem into the computation of mode specific functions $\mathbf{g}_{\mathbf{c}}(\tau, \mathbf{w})$ and the enumerative definition for the dynamic response in (23).

For contact mode ambiguities, we present a method in Section III-G.1 to identify sets of control inputs which are guaranteed to unambiguously achieve a desired contact mode. This can be particularly useful for tasks which critically rely on the realization of particular modes, such as during climbing with a snake robot by ensuring that there is a set of supporting contacts which are fixed relative to the environment.

For ambiguities within a contact mode, the conditions provided in Section III-D do not necessarily result in ambiguous reaction forces and accelerations in all cases. In 
Section III-G.2, we provide certain conditions for the degeneracy of the polytope describing the domains of mode specific dynamic response functions that are necessary for concluding the presence of such ambiguities. We also give a number of illustrative examples to give some intuition towards possible causes of multiplicity in force and acceleration solutions.

\section{G.1 Contact Mode Ambiguities}

The algorithm presented in Section III-E computes the set, $\mathcal{T}_{\mathbf{c}}$, of control inputs consistent with the constraints of a given contact mode. Clearly, non-empty intersections between the domains of different contact modes indicates multiplicity of solutions for certain regions in the space of control inputs. The result is a contact mode ambiguity, wherein the rigid model alone is not sufficient to determine which contact mode will be physically realized for certain control inputs. For many applications, it is important to identify and stay away from such ambiguities by avoiding control inputs for which the model is unable to yield a unique solution. This section presents a simple algorithm to compute unambiguous domains for each contact mode.

Consider a particular pair of contact modes $\mathbf{c}_{i}$ and $\mathbf{c}_{j}$. Control inputs for which there is an ambiguity between these modes can be represented by the intersection of the corresponding domains, $\mathcal{T}_{\mathbf{c}_{i}} \cap \mathcal{T}_{\mathbf{c}_{j}}$. This intersection is easily computed by taking the union of the associated linear inequality systems. Similar to our reduction of domains in Section III-E, all non-supporting inequalities can be eliminated through linear programming to produce a simple representation of the set.

Once all such pairwise intersections are computed, the unambiguous domain $\mathcal{T}_{\mathbf{c}}{ }^{*}$ for a contact mode $\mathbf{c}_{i}$ is defined as

$$
\mathcal{T}_{\mathbf{c}}^{*}:=\mathcal{T}_{\mathbf{c}}-\bigcup_{\overline{\mathbf{c}} \in\left(\mathcal{M}_{p}-\mathbf{c}\right)}\left(\mathcal{T}_{\mathbf{c}} \cap \mathcal{T}_{\overline{\mathbf{c}}}\right)
$$

which will, in general, be a non-convex volume in the space of control inputs.

For instance, in our two-link example, large values of the coefficients of friction $\mu$ result in unbounded ranges for the ambiguity variables. This makes the fixed contact mode feasible for a very large range of control inputs. On the other hand, small control inputs also allow for sliding contact modes due to the insufficient frictional forces that fail to balance the action of gravity. As a consequence, there are control torques for which it is impossible to determine the immobilization of the system from the rigid model alone. Properly designed controllers must stay away from such regions.

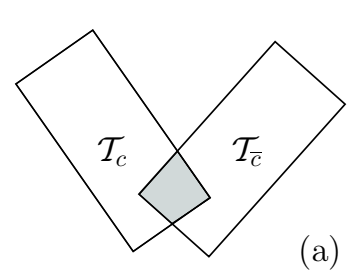

(a)
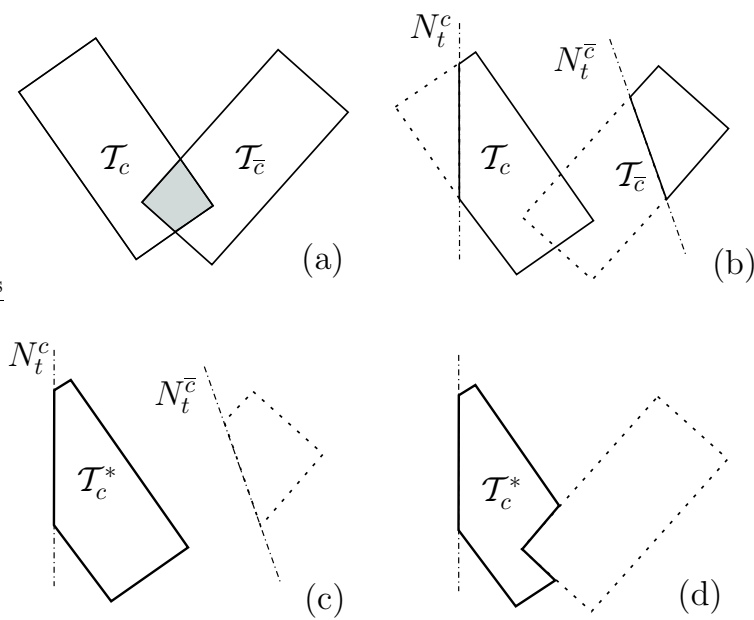

(c)

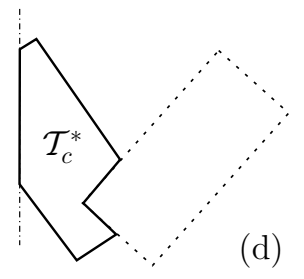

(d)
Fig. 4. Interaction of task constraints with mode ambiguities. (a) Domains for contact modes $\mathbf{c}$ and $\overline{\mathbf{c}}$ with no task constraints imposed. Inputs in the shaded region will result in a mode ambiguity. (b) Domains after applying task constraints. The same task constraint may have different effects $N_{t}^{c}$ and $N_{t}^{\bar{c}}$ in two different modes. (c) Imposing task constraints prior to computing unambiguous domain $\mathcal{T}_{\mathbf{c}}^{*}$ results in improper inclusion of ambiguous region. (d) Properly computed unambiguous domain $\mathcal{T}_{\mathbf{c}}^{*}$.

An important aspect of computing $\mathcal{T}_{\mathbf{c}}{ }^{*}$ is the consideration of task constraints. These constraints can be very useful in the design of controllers by reducing the domains for each mode to achieve some additional, desired properties of associated solutions, such as bounded reaction forces. However, the impact of the task constraints $N_{t}$ on the domain $\mathcal{T}_{\mathbf{c}}$ is usually different for each contact mode $c$ and this difference may interfere with proper identification of unambiguous domains.

Consider, for example, a scenario similar to the two-link example above, where there is a physical ambiguity betwen the fixed contact mode $\mathbf{c}$ and the sliding contact mode $\overline{\mathbf{c}}$ (shaded region in Figure 4.a). As illustrated in Figures 4.b and 4.c, the consideration of task constraints prior to elimi- 
nating domain intersections results in improperly including this ambiguous region in the unambiguous domain of the fixed mode. In order to eliminate this problem, the domain intersections of (34) must be performed prior to applying task constraints (see Figure 4.d).

\section{G.2 Ambiguities within a particular contact mode}

Whenever $\mathbf{A}$ in the equality constraints of (25) does not have full column rank, force and acceleration ambiguities may occur within a contact mode. In such cases, we parameterize the set of possible solutions with a vector of ambiguity variables, w, whose ranges are also restricted through the computation of the feasibility domain $\mathcal{D}_{\mathbf{c}}$.

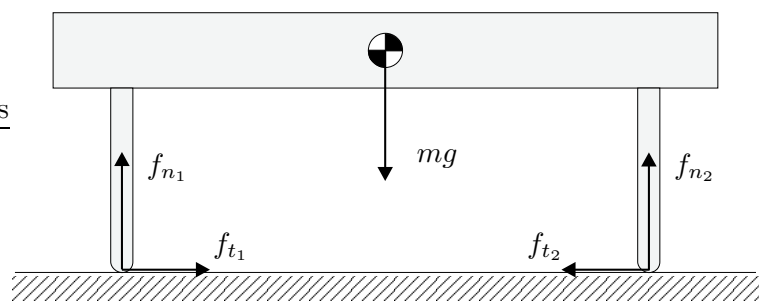

Fig. 5. A rigid table with indeterminate tangential forces

Figure 5 illustrates an example where a rigid planar table with two legs is positioned on a flat floor. If the contact points are assumed to be fixed, then the matrix A takes the form

$$
\mathbf{A}=\left[\begin{array}{cc}
\mathbf{J} & \mathbf{0} \\
\mathbf{M}(\mathbf{q}) & -\mathbf{J}^{T}
\end{array}\right]
$$

where $\mathbf{M}(\mathbf{q}) \in \mathbb{R}^{3 \times 3}$ and $\mathbf{J} \in \mathbb{R}^{4 \times 3}$. Due to the rank deficiency of $\mathbf{J}^{T}$, A has a one dimensional null-space. This corresponds to unknown opposing tangential forces on the legs. Even though the accelerations are uniquely determined with $\ddot{\mathbf{q}}=0$, there is ambiguity in the tangential contact reaction forces.

Similarly, Figure 6 portrays a planar ball bearing in contact with four flat surfaces, illustrating a situation wherein there is a coupled ambiguity in computing both the reaction forces and accelerations. Under the assumption that all contact points are sliding, we are once again left with a rank deficiency in the matrix $\mathbf{A}$. The result is unknown normal reaction forces parameterized by ambiguity variables $\mathbf{w}$. The result of this ambiguity of compressive force

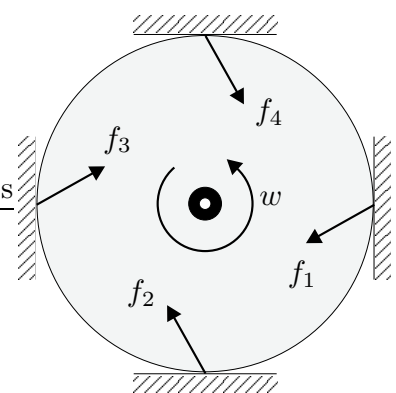

Fig. 6. Ball bearing with sliding contacts and indeterminate acceleration

on the ball is an ambiguous torsional load on the ball and hence non-unique solutions for the rotational acceleration of the ball as well as reaction force.

These two examples also illustrate that ambiguities within a contact mode may be purely in reaction forces, or in both system accelerations and reaction forces. Further analysis on the structure of $\mathbf{N}(\mathbf{A})$ as defined in Section III$\mathrm{D}$ would be necessary for any conclusions in this context. For certain applications, such as the maximal thrust controller for flipping, it is important to establish the uniqueness of solutions in accelerations while ambiguities in reaction forces can be tolerated.

Finally, a necessary condition for the presence of ambiguities within a mode is the non-degeneracy of the feasible domain $\mathcal{D}_{\mathbf{c}}$. In cases where the polytope representation for this domain collapses in the direction of the ambiguity variables, there will be no ambiguities even though the form of $\mathbf{g}_{\mathbf{c}}(\tau, \mathbf{w})$ explicitly incorporates the vector $\mathbf{w}$. Note, however, that this corresponds to a very fragile situation where even small changes in parameters may result in the expansion of the domain back into a non-degenerate volume, reintroducing multiple solutions.

\section{H. Computational Complexity}

In this section, we report on the average time complexity of our algorithm, particularly focusing on its dependence on the number of contacts $k$, in order to characterize its practical applicability.

We present the complexity analysis of our algorithm in two components: Solving for the dynamic response within a contact mode and the enumeration of feasible modes. Not 
surprisingly, the dominant complexity arises from the contact mode enumeration and is exponential in the number of contacts $k$ as well as the dimension of the space ambiguities within a contact mode, $w$. Nevertheless, the algorithm complexity scales as a cubic polynomial in the number of coordinates $q$ and the number of control inputs $t$.

\section{H.1 Mode-Specific Dynamic Response and Domain}

In the absence of external constraints, solving the linear system in (25) for $\mathbf{g}_{\mathbf{c}}$ requires the Singular Value Decomposition (SVD) of a matrix with dimensions $(q+2 k) \times(q+2 k)$. When $\mathbf{A}$ is not full row rank, an additional step is needed to find the pseudo-inverse of a matrix with dimensions $(q+2 k) \times(q+2 k+t)$, resulting in an overall complexity of $O\left((q+2 k+t)^{3}\right)$, or cubic in the largest dimension of the matrix [8]. We specifically focus on the complexity with respect to the number of contacts, yielding $O\left(k^{3}\right)$ for the solution of all equality constraints.

In solving for the domain associated with a particular contact mode, two inequality constraints for each contact must be considered in the worst case. The resulting matrix $\mathbf{N}$ thus has dimensions $(2 k) \times(t+w)$. The elimination of non-supporting hyperplanes from this system through the Simplex algorithm for solving linear programs is known to have an exponential worst case running time. However, for our analysis, we use the expected running time of the algorithm, which is only linear in the number of constraints [34]. Since a linear program must be solved for each of the $2 k$ inequality constraints, the expected running time for this step, once again focusing on $k$, is $O\left(k^{2}\right)$.

In cases where there ar ambiguities within a mode, polytope projection is performed. The Fourier-Motzkin projection technique is known to produce many spurious inequality constraints, with an upper bound of $O\left(\mathrm{~m}^{2}\right)$ for the number of resulting constraints, where $m$ is the number of inequalities prior to the projection [15]. Starting with $2 k$ constraints, $w$ projections are needed, resulting in a complexity of $O\left(k^{2 w}\right)$ which is exponential in the number of ambiguity variables. Finally eliminating non-supporting hyperplanes from this system through linear programming, results in a total expected running time for this step as
$O\left(k^{4 w}\right)$

\section{H.2 Enumeration of Feasible Contact Modes}

The number of possible contact modes for a multi-rigidbody system is $4^{k}$. The worst case is when all contact points are stationary, forcing our algorithm to consider all possible modes. In contrast, the best case is when all contacts have nonzero velocities, resulting in reductions in feasible contact modes to $2^{k}$. Both cases, are exponential in the number of contacts.

Each feasible mode requires the computation of the mode specific dynamic response, whose average complexity was presented in the previous section. The overall complexity for our algorithm hence becomes $O\left(2^{k}\left(k^{3}+k^{4 w}\right)\right)$, which naturally preserves the exponential complexity resulting from the mode enumeration.

Despite certain improvements resulting from the analysis of kinematic feasibility to improve on the exponential running time, the general problem of determining contact modes is generally known to be exponential [4]. Even though some algorithms such as Lemke's [16] are widely used in building multi-rigid-body simulations, and are capable of finding single solutions without the exponential complexity, they sacrifice completeness and the ability to accurately identify ambiguities, which are critical components for the problems we are interested in. The complexity of our proposed methods is not significantly worse than currently known algorithms for accurate modeling of multirigid-body systems under frictional contact. Even though our ability to produce concise representations of feasible sets of control inputs for any given contact mode induces additional, potentially exponential, elements in the overall complexity of our algorithm, we believe that their utility in the design of model-based controller is still valuable.

\section{Dynamic Flipping with RHex}

Design and analysis of dynamic robot behaviors with multiple frictional contacts is a challenging problem. Two of the authors previously investigated an example of such a task - dynamical recovery of correct body orientation for a hexapedal robot [30]. However, the "blind", model-based controller design in this previous approach makes very rigid 
assumptions about the contact modes on which the underlying models are based. Furthermore, the absence of explicit constraints to ensure that all assumed contact modes are realized, results in the overall inaccuracy of the models and dimished performance of the resulting controller.

In this section we apply the general modeling tool for dynamical systems with multiple contacts developed in preceding sections to build a dynamical flipping controller for a planar hexapod. Our controller design is very close in principle to the method described in [30]. However, in our new approach, we do not make rigid assumptions regarding contact modes, ensuring the validity of the resulting strategy on a much larger set of states.

We also introduce a more complex, compliant model that is unsuitable for the design of model-based controllers, but provides a physically accurate benchmark for the characterization of the newly introduced controller. Due to the limitations of our current experimental platform, we are currently not able to deploy the new controller on RHex and hence use the simulated compliant model as a basis for comparison between different model-based flipping controllers.

\section{A. Overview of Previous Models and Controllers}

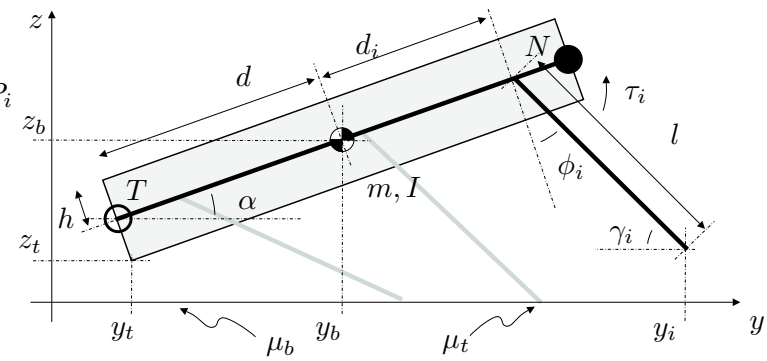

Fig. 7. Generic 3DOF planar flipping model

Figure 7 illustrates a generic planar flipping model whose constrained instances constitute the basis for the controller designs in [30]. Three massless rigid legs - each representing a pair of RHex's legs - are attached to a rectangular rigid body with mass $m$ and inertia $I$. The attachment points of the legs are fixed at $d_{i}$, along the midline of the rectangular body. This line also defines the orientation of the body, $\alpha$, with respect to the horizontal. The body length and height are $2 d$ and $2 h$, respectively. the bodyground and toe-ground contacts are assumed to experience Coulomb friction with coefficients $\mu_{b}$ and $\mu_{t}$, respectively.

All flipping controllers for RHex adopt the same general structure. Starting from a stationary position on the ground, the robot quickly thrusts itself upward while maintaining contact between the ground and the endpoint of its body as the front and middle legs successively leave the ground. Depending on the frictional properties of the ground contacts, this thrust generates body kinetic energy that may in some cases be sufficient for a successful flip.

Not surprisingly, the performance of the flipping controller is predominantly determined by the amount of energy that can be injected into the system through the thrust phase. As a consequence, our focus in this paper is on thrust controllers that are based on sufficiently accurate models of system dynamics under Coulomb frictional contact. In using such models, the blind controller design in [30] was forced to consider only a small number of contact modes due to the absence of a general tool to uniformly handle the set of all possible equations of motion under different contact conditions. The choice of particular contact modes to be modeled was primarily motivated by empirical studies on open-loop flipping algorithms. In particular, two constrained models were used. The first model was derived under the assumption that the front and middle legs slide backwards whereas the body and the rear legs slide forward, all maintaining contact with the ground throughout flipping. In contrast, the second model assumed that the rear toes were stuck on the ground with the body sliding forward and the middle and front legs sliding backwards. These two particular models were chosen primarily based on empirical observations of flipping experiments, the majority of which exhibited initial backward sliding of the rear toe followed by stiction towards the end of each flip. Due to the highly empirical nature of contact mode estimation, we term the resulting controller the "blind" model-based flipping controller. In contrast, the general method described in Section III uniformly considers all kinematically feasible contact modes, ensuring sound and complete identification of all friction related anomalies in the model. 


\section{B. Current Model and Extensions}

\section{B.1 Lagrangian Formulation of the Dynamics}

The general planar model illustrated in Figure 7 also underlies our current design for model based flipping controllers. However, in contast to the Newton-Euler formulation adopted in [30], we present a Lagrangian formulation of the same dynamical system as an instance of the general framework of Section III-A.

We will find it convenient to work in the generalized coordinates $\mathbf{q}:=\left[y_{b}, z_{b}, \alpha\right]^{T}$ for the unconstrained planar system. The dynamics are those of a simple planar rigid body under external force and torque inputs, and take the form

$$
\left[\begin{array}{ccc}
m & 0 & 0 \\
0 & m & 0 \\
0 & 0 & I
\end{array}\right] \ddot{\mathbf{q}}+\left[\begin{array}{c}
0 \\
-m g \\
0
\end{array}\right]=\left(D_{\mathbf{q}} \mathbf{x}\right)^{T} \mathbf{f},
$$

where the jacobian of contact coordinates with respect to model coordinates is derived with the hip angles $\phi_{i}$ kept fixed. In contrast to (5), the control inputs in this case do not directly act on the generalized coordinates but are rather applied to the hip angle coordinates $\phi_{i}$. As we assume all legs to be massless, these hip torque inputs are incorporated into the equations of motion as algebraic equality constraints on the contact reaction forces, taking the form

$$
\left(D_{\phi} \mathbf{x}\right)^{T} \mathbf{f}=\tau .
$$

Given a contact mode vector $\mathbf{c}$ and a control input torque vector $\tau$, the equations of motion and contact reaction forces can then be found by solving (35) under the constraints (36) as well as the contact mode constraints of Section III-B.

\section{B.2 Maximal Thrust Across All Contact Modes}

As outlined in Section IV-A, the main challenge in the design of flipping controllers is to inject as much kinetic energy into the pitch degree of freedom as possible during the thrust phase. Although not "optimal" in a formal sense - the translational degree of freedom if the body is not considered in the optimization, this approach effectively targets the task at hand: maximal pitch acceleration of the body to overcome the potential energy barrier of the vertical configuration.

In the absence of contact constraints with frictional nonlinearities, obtaining maximal thrust for the resulting purely continuous system would have been as simple as solving a single linear programming problem instance to maximize $\ddot{\alpha}$. However, the flipping behavior critically relies on frictional contact interactions with the environment, causing solutions for system accelerations to be piecewise linear in the control inputs. Each possible contact mode must now be individually considered, together with the additional constraints it imposes on the feasible set of torques. In light of these observations, our controller design attempts to be as general as possible by considering all possible contact modes, but conservative enough to ensure that the physical system is guaranteed to yield the predicted response.

Our controller enumerates all possible contact modes and solves a linear programming problem to maximize the pitch acceleration $\ddot{\alpha}$, subject to external constraints on control inputs - such as motor torque limitations - as well as the feasibility constraints of Section III-E arising from the assumed contact mode. Formally, given the current state $(\mathbf{q}, \dot{\mathbf{q}})$ and a particular contact mode $\mathbf{c}$ which is kinematically feasible, we compute control inputs to yield the maximal pitch acceleration response as

$$
\begin{aligned}
\tau_{\mathbf{c}}^{*} & :=\underset{\tau \in \mathcal{T}_{\mathbf{c}}}{\operatorname{argmax}}\left(\ddot{\alpha}_{\mathbf{c}}(\tau)\right) \\
\text { and } \quad \ddot{\alpha}_{\mathbf{c}}^{*} & :=\ddot{\alpha}_{\mathbf{c}}\left(\tau_{\mathbf{c}}^{*}\right),
\end{aligned}
$$

where $\mathcal{T}_{\mathbf{c}}$ denotes the set of feasible torques as described in Section III-E.

In constraining $\mathcal{T}_{\mathbf{c}}$, we only consider regions of the torque space that unambiguously guarantee the contact mode $\mathbf{c}$, using the methods described in Section III-G.1. In addition, we impose a task constraint such that the normal component of the ground reaction force is always kept greater than a manually chosen threshold to ensure continued contact of the body with the ground, even in the presence of model inaccuracies and sensor noise ${ }^{6}$.

\footnotetext{
${ }^{6}$ Motivations for this constraint are detailed in [30]
} 
Following this formulation, we can consider all the kinematically feasible contact modes in the set $\mathcal{M}_{f}$, yielding the solution to the global problem as

$$
\begin{aligned}
\mathbf{c}^{*} & :=\underset{\mathbf{c} \in \mathcal{M}_{f}}{\operatorname{argmax}}\left(\ddot{\alpha}_{\mathbf{c}}^{*}\right) \\
\tau & =\tau_{\mathbf{c}^{*}}^{*} .
\end{aligned}
$$

\section{Simulation Studies}

The blind controller used in [30] was successfully deployed on RHex and extensive experimental evidence was given to establish the performance and prediction accuracy of underlying models. RHex's limited sensor suite - an onboard fiber-optic gyro - currently only yields body orientation measurements and cannot supply translational coordinates and velocities of its body. Practical deployment of the blind controller was made possible by a number of assumptions and simplifications, reducing the state dependence of model equations to only the pitch degree of freedom in all cases. However, the current controller is based on a more complete formulation of contact modes and the associated dynamics does not admit similar simplifications. Furthermore, the current implementations with the general method require significantly more computational resources and are not feasible for practical deployment at this time.

Consequently, in this paper, we use simulation studies to illustrate the accuracy and robustness of the new approach and present comparisons to simulated versions of the blind controller. In doing so, we also present evidence to ensure the validity of our simulation environment with respect to previous flipping experiments on RHex.

\section{C.1 Simulation Environment}

In this section, we describe a more complex, compliant planar model that underlies all our simulation results and verify its accuracy with respect to the physical robot. Even though this compliant model is superior to the simpler rigid models in its predictive performance, its analytical inversion is not tractable, making it unsuitable for the design of model-based flipping controllers. Furthermore, our eventual goal of online deployment of resulting controller designs precludes the possibility of numerical optimization over forward integration of this compliant model due to high computational costs associated with such approaches. Our main motivation in introducing this model is to provide an accurate benchmark for the characterization of the new controller design.

The planar compliant model is morphologically similar to the model presented in Section IV-A but differs in three major aspects. First, all three legs incorporate radial compliance and damping in contrast to the rigid leg links on the model we use to design our controllers. This enables us to more accurately capture RHex's behavior wherein the compliance in the legs has significant impact on behavior in certain configurations.

Second, we model the feet independently by incorporating small point masses on the toes. Forces transmitted through the leg, frictional forces and gravity act on the toes and govern their motion. Both the non-rigid coupling between the toes and the body as well as the lack of rotational inertia of the toes allows us to avoid ambiguities and inconsistencies that are typical of frictional Coulomb contacts, and hence yield a reliable simulation environment free of singularities.

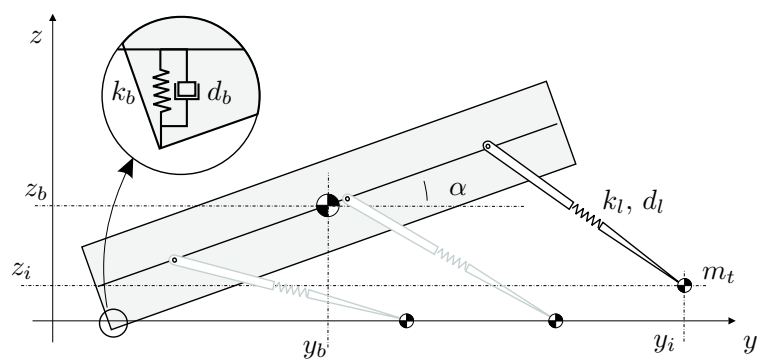

Fig. 8. Simulated planar flipping model with compliant legs

Finally, we adopt a penetration based model for the body-ground contact to eliminate the need for explicit modeling of impacts as well as Coulomb model induced anomalies such as Painlevé's problem. The normal ground reaction force is computed as a function of body penetration distance and velocity, whereas the horizontal force adopts a pure Coulomb model. This extension also has the benefit of approximating the padded protective skin on RHex whose deformations are most likely manifested in experiments as deviations from the rigid model and inaccuracies in the kinematic assumptions of state estimators. 


\begin{tabular}{|r||r||c||c|}
\hline$d$ & $0.25 \mathrm{~m}$ & $m$ & $8.5 \mathrm{~kg}$ \\
\hline$d_{1}$ & $-0.19 \mathrm{~m}$ & $I$ & $0.144 \mathrm{kgm}^{2}$ \\
\hline$d_{2}$ & $0.015 \mathrm{~m}$ & $m_{t}$ & $0.05 \mathrm{~kg}$ \\
\hline$d_{3}$ & $0.22 \mathrm{~m}$ & $k_{b}$ & $10000 \mathrm{~N} / \mathrm{m}$ \\
\hline$h$ & $0.05 \mathrm{~m}$ & $d_{b}$ & $2000 \mathrm{Ns} / \mathrm{m}$ \\
\hline$l$ & $0.17 \mathrm{~m}$ & $k_{l}$ & $5000 \mathrm{~N} / \mathrm{m}$ \\
\hline & & $d_{l}$ & $500 \mathrm{Ns} / \mathrm{m}$ \\
\hline
\end{tabular}

TABLE IV

KINEMATIC AND DYNAMIC PARAMETERS FOR ALL FLIPPING SIMULATIONS

Figure 8 together with the kinematic and dynamic parameters in Table IV specify the model used in our simulation studies. The scripts, code and data files in Extensions 6 and 7 can be used to reproduce all the simulation results and the associated plots presented in this paper.
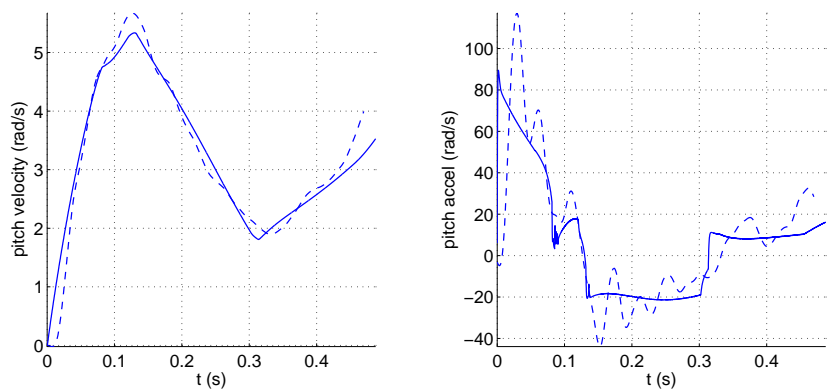

Fig. 9. Validation of simulation accuracy with respect to experiments on RHex. Solid lines show simulated flipping, whereas dashed lines are experimental measurements of RHex flipping on linoleum, both under the action of the old, model-based controller with $\mu_{t}=0.39$.

Figure 9 illustrates a comparison between a flipping simulation with the compliant model described in this section and an example flipping experiment on RHex (see Extension 1), both under the action of the blind model-based controller described in [30]. Most of the differences arise from the torsional compliance of RHex's legs which introduce significant delay in the transmission of motor torque to the body. In contrast to simulations presented in [30] which directly use the rigid model, the compliant model with toe masses more accurately simulates the early liftoff of the legs as well as the true stiction behavior of the rear toe.

\section{C.2 Performance of Previous Controllers}

In this section, we present simulation results with the blind controller that illustrate cases in which its rigid assumptions regarding contact modes are inaccurate. In particular, we show that the performance of the indirect detection of rear toe stiction based on measurements of pitch acceleration largely depends on the closeness of surface parameters to empiricial tuning conditions. In contrast, the new controller inherently handles contact mode induced switching of models and does not rely on an empirically tuned filter.

Figure 10 illustrates the baseline performance of the blind controller on linoleum, characterized by a relatively low friction coefficient at $\mu_{t}=0.39$. This corresponds to the conditions in which the toe stiction detection algorithm was tuned and hence yields relatively accurate timing for the switching of models.

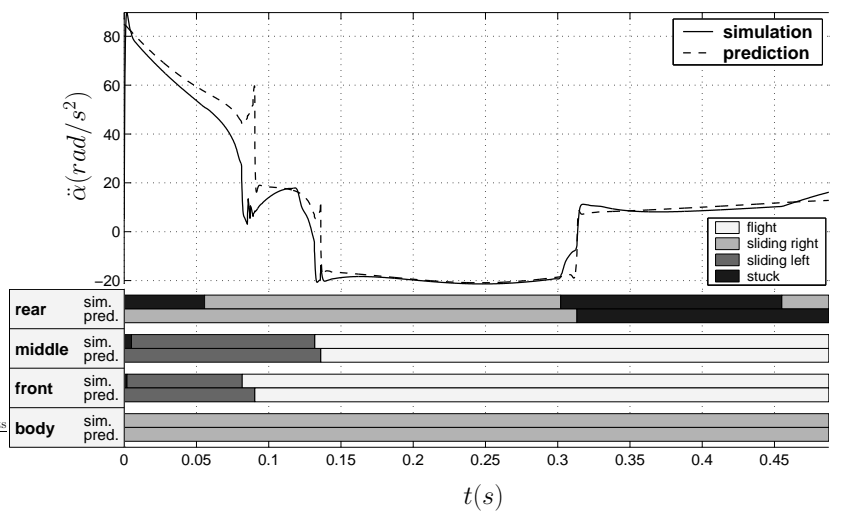

Fig. 10. Flipping with the blind model-based controller with low toe friction $\mu_{t}=0.39$. Top half illustrates model prediction and the actual simulation data for the pitch acceleration, whereas the bottom half portrays the progression of contact modes for all the legs and the body. See Extension 2 for the associated animation.

In contrast, Figure 11 illustrates the blind controller on a surface with higher coefficient of friction such as asphalt with $\mu_{t}=0.6$. In this case, the rise in the pitch acceleration due to the stiction of the rear toe is not sufficient to trigger the model switch in a timely manner. Instead, the controller maintains a wrong assumption about the contact 
mode until the body-ground contact comes to a halt much later, inducing the necessary sharp change in the pitch acceleration. The consequent delay in the switch to the model with stuck rear toe usually results in degraded thrust performance as the sliding toe model is more conservative and applies much lower torque actuation to the rear leg.

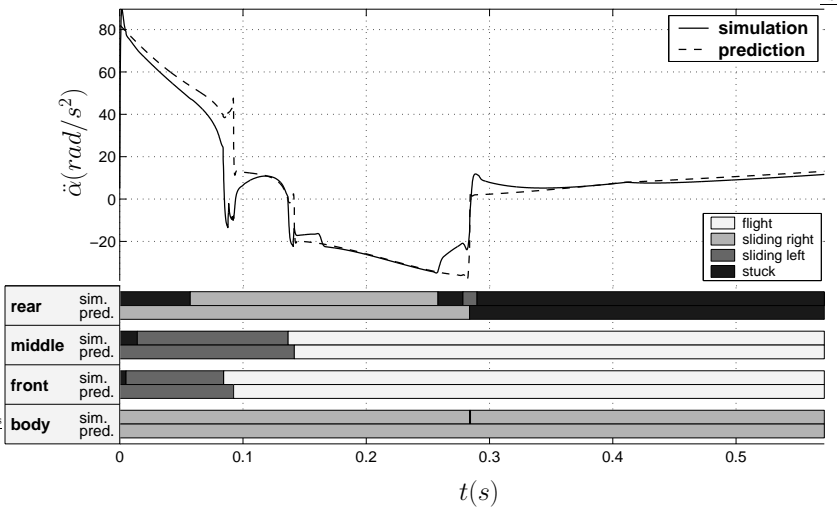

Fig. 11. Flipping with the blind model-based controller with high toe friction $\mu_{t}=0.6$. See Extension 3 for the associated animation.

\section{C.3 Coping with Model Inaccuracies}

Section III-F describes a step in our contact modeling algorithm which permits computationally efficient elimination of contact modes that are physically impossible based on the kinematic maps (3) and (4) for contact velocities and accelerations. Not surprisingly, this procedure is very sensitive to errors in the kinematic model with respect to the physical system under consideration and can lead to erroneous elimination of some contact modes. In practice, equality conditions on components of contact velocities are almost always guaranteed to fail and membership tests against intervals around the desired quantities is a much more robust method against inevitable model inaccuracies and sensor noise. Our controller design and the optimization across feasible contact modes presented in Section IVB.2 is particularly vulnerable to such errors which can lead to sub-maximal thrust solutions and controller chatter.

Our approach is to relax the conditions in Table III-F to prevent overly aggressive elimination of feasible contact modes. We introduce thresholds $v_{i}^{0}$ on the velocities of each toe such that whenever $\left|\dot{x}_{t_{i}}\right|<v_{i}^{0}$, we refrain from eliminating any modes for that contact. Our choice of $v_{i}^{0}$ is based on
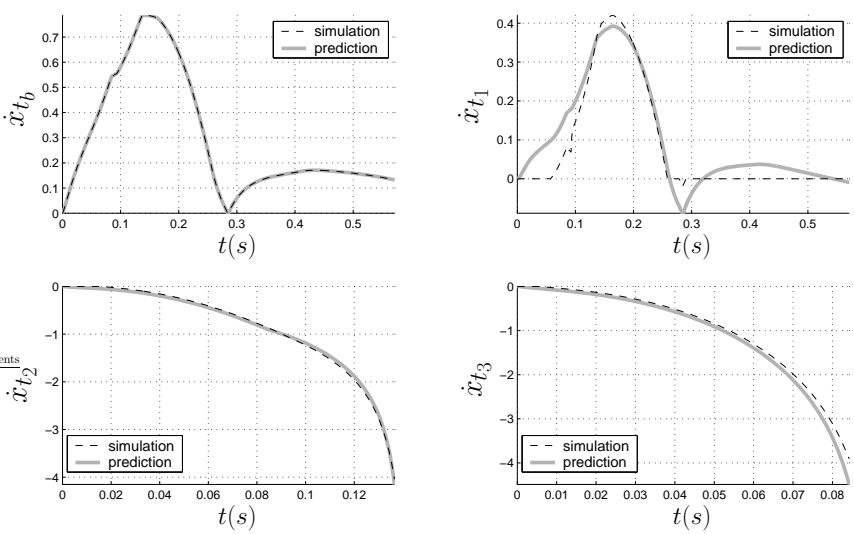

Fig. 12. Rigid model predictions of horizontal contact velocities compared with simulation trajectories

simulations to identify the relative inaccuracy of the rigid flipping model of Section IV-A with respect to the simulated model with leg compliance and body penetration described in Section IV-C.1. Figure 12 illustrates rigid model predictions of toe velocities against simulated toe velocities through a typical flipping run with the blind controller. In order to determine the thresholds $v_{i}^{0}$ for toe and body contacts, we consider intervals wherein the contact is fixed in simulation and identify the range of model predictions for velocities. All flipping experiments reported in [30] as well as simulations of the blind and new model-based controllers yield trajectories that are kinematically very similar. As a consequence, we use the thresholds $v_{1}^{0}=0.08, v_{2}^{0}=0.001$ and $v_{3}^{0}=0.001$ recovered from Figure 12 for all simulations with our new controller.

\section{C.4 Performance of the New Controller}

As described in Section IV-B.2, the application of the general method for contact mode analysis to the design of a flipping controller allows the consideration of all kinematically feasible possibilities in finding the maximal thrust torque. As a consequence, the new controller does not need to make any task specific assumptions on the progression of body or leg contact states and hence has a much larger domain of validity with respect to variations in surface conditions.

Even compared to the blind controller operating under the low friction conditions of Figure 10 for which it was 
tuned, Figure 13 illustrates that the new controller's estimate of contact modes is much more accurate. Notably, it was even able to match the renewed right sliding of the rear toe towards the end of the flip around $t=0.45 \mathrm{~s}$.

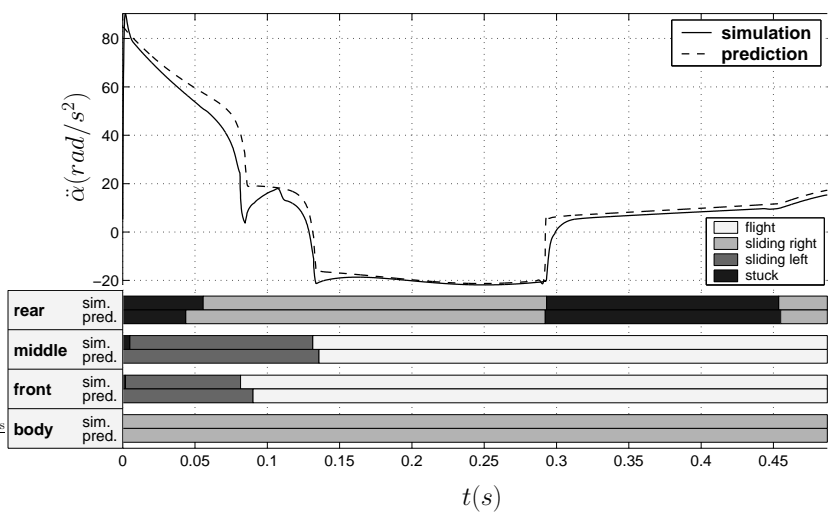

Fig. 13. Flipping with the new controller with low toe friction $\mu_{t}=$ 0.39. See Extension 4 for the associated animation.

The robustness of the new controller is much more evident on high friction surfaces as illustrated in Figure 14. In contrast to the poor predictive performance of the blind controller in Figure 11, the new controller is able to find much more accurately the point in which the stiction of the rear toe yields the maximal thrust and appropriately adjusts the hip torques. As a result, the resulting thrust phase is more effective with a $8 \%$ improvement in the time it takes to complete a flip (see Figure 15).

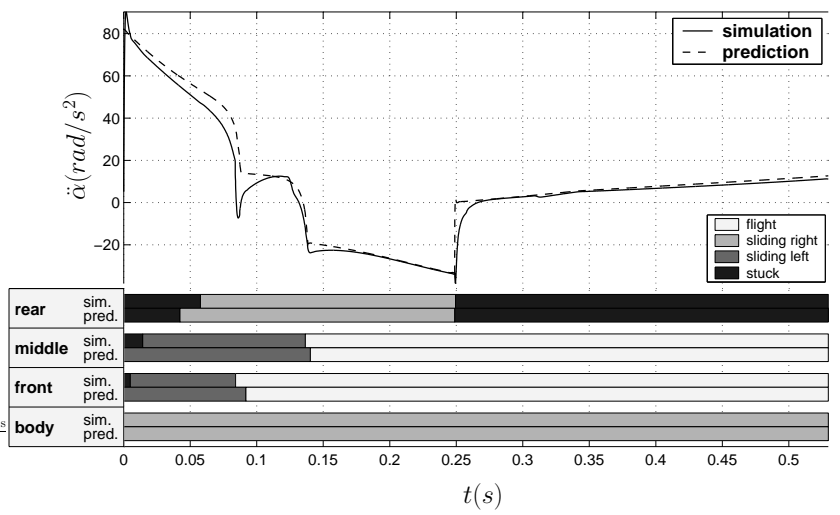

Fig. 14. Flipping with the new controller with high toe friction $\mu_{t}=0.6$. See Extension 5 for the associated animation.

Finally, we compare the accuracies of the blind and new models through the mean square error in their pitch accel-

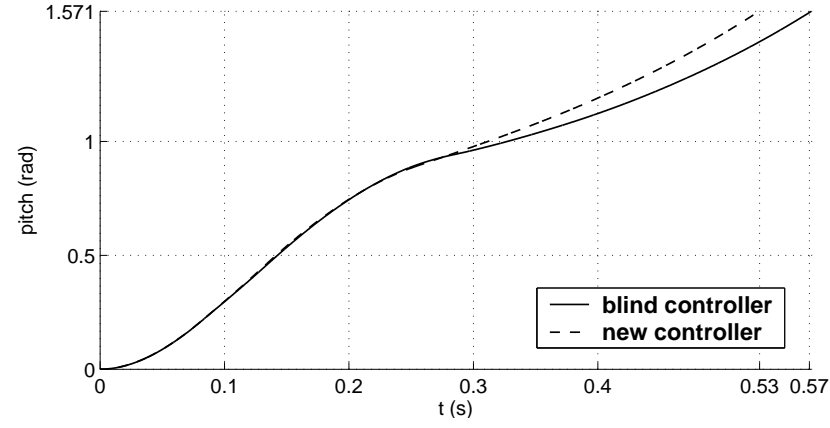

Fig. 15. Time trajectories of the pitch during flipping with the blind controller (solid) and the new controller (dashed) on a high friction surface $\left(\mu_{t}=0.6\right)$. The new controller is roughly $8 \%$ quicker to complete a flip than the blind controller.

\begin{tabular}{|r|c|c|}
\hline & $\mu_{t}=0.39$ & $\mu_{t}=0.6$ \\
\hline \hline blind & 11.22 & 11.56 \\
\hline new & 6.65 & 6.68 \\
\hline
\end{tabular}

TABLE V

ROOT MEAN SQUARE ERROR IN PITCH ACCELERATION PREDICTIONS OF the BLind AND the NEW CONTROllers. ERror units ARe $\mathrm{rad} / \mathrm{s}^{2}$

eration predictions, computed as

$$
R M S E:=\left(\frac{1}{t_{f}} \int_{0}^{t_{f}}(\hat{\ddot{\alpha}}(t)-\ddot{\alpha}(t))^{2} d t\right)^{1 / 2} .
$$

Table $\mathrm{V}$ summarizes the prediction performance of both controllers on the low and high friction surfaces. The new controller and the underlying model has significantly better predictive accuracy than the old, blind controller.

\section{Conclusion and Future Work}

\section{A. Conclusion}

Control of robotic systems subject to frictional constraints can be a challenge due to the non-linearities and physical modeling challenges inherent in modeling frictional contact. In this paper we model such systems as multi-rigid-bodies subject to coulomb friction and linear complementarity constraints.

We develop a computational framework to compute the instantaneous dynamic response of such systems as a function of the control torque. The algorithm developed in 
particular characterizes all possible dynamic solutions of the system, thus allowing for identification of areas of ambiguity in the dynamic response. Our algorithm decomposes the dynamic response by contact mode, thus dividing the computation of the system dynamics into linear sub-problems.

Based on the computed instantaneous dynamic response, a controller is generated for a flipping application which optimizes the system behavior with respect to the instantaneous dynamics, in particular maximizing the pitch acceleration subject to additional linear constraints.

\section{B. Future Work}

An obvious extension to the work is a treatment of impact, which may be a crucial aspect of controlling some robotic systems subject to frictional contact. Of course, the extension to three dimensions is also important, and will come with increased computational cost. Since the coefficient of friction is often not well known, or even constant as a function of time, an extension of these control techniques with some measure of provable robustness to variations in friction coefficient, and possibly other kinematic or dynamic quantities, would be an important extension.

Practically, the issue of inexact or incomplete state measurement becomes important when applying this control method on actual robot systems. In particular, the prediction of contact point velocities based on incomplete or noisy sensor measurements is crucial, especially when the contact point velocity is low, since the model dynamics undergoes a change of mode on the measure zero set of the sticking contact point.

\section{ACKNOWLEDGMENT}

The authors would like to thank Devin Balkcom, Siddhartha Srinivasa, and Howie Choset for comments on the algorithm. This work was supported in part by ARO grant DAAD19-02-1-0383 and DARPA grants DARPA/SPAWAR N66001-03-C-8045, DARPA/ONR N00014-98-1-0747 and DARPA/SPAWAR N66001-00-C-8026.

\section{Index to Multimedia Extensions}

Extension 1. Video: LinoleumSingleShot.mpg. High speed video (150fps) of RHex 1.5 flipping on linoleum with the blind controller.

Extension 2. Video: blind_0.39.mpg. Slow motion animation of a planar flipping simulation with the blind controller on a surface with $\mu_{t}=0.39$

Extension 3. Video: blind_0.6.mpg. Slow motion animation of a planar flipping simulation with the blind controller on a surface with $\mu_{t}=0.6$

Extension 4. Video: improved_0.39.mpg. Slow motion animation of a planar flipping simulation with the improved controller on a surface with $\mu_{t}=0.39$

Extension 5. Video: improved_0.6.mpg. Slow motion animation of a planar flipping simulation with the improved controller on a surface with $\mu_{t}=0.6$

Extension 6. Data: data_scripts.tar.gz. Data files and visualization scripts for all the experiments and simulations. Please see README.txt in this archive for details on the format of data files and the usage of associated scripts.

Extension 7. Code: code.tar.gz. Simulation code used to generate all data in the paper. Please see README.txt in this archive for details on how to compile and use the simulator.

\section{REFERENCES}

[1] M. Anitescu, "On solving mathematical programs with complementarity constraints as nonlinear programs," Tech. Rep. ANL/MCS-P864-1200, Argonne National Laboratory, Argonne, IL., 2000.

[2] M. Anitescu and F. A. Potra, "Formulating dynamic multi-rigidbody contact problems with friction as solvable linear complementarity problems," Nonlinear Dynamics, vol. 14, no. 3, pp. 231-247, November 1997.

[3] D. Balkcom and J.C. Trinkle, "Computing wrench cones for planar rigid body contact tasks," International Journal Of Robotics Research, vol. 21, no. 12, pp. 1053-1066, December 2002.

[4] D. Baraff, "Determining frictional inconsistency is NPcomplete," Tech. Rep. TR90-1112, Computer Science Department, Cornell University, April 1990.

[5] T. Bretl, S. Rock, and J. C. Latombe, "Motion planning for a three-limbed climbing robot in vertical natural terrain using fast identification," in Proc. IEEE Int. Conf. on Robotics and Automation, 2003, pp. 3964-3969.

[6] B. Brogliato, "Some perspectives on the analysis and control of complementarity systems," IEEE Transactions on Automatic Control, vol. 48, no. 6, pp. 918-935, June 2003.

[7] H. Choset and W. Henning, "A follow-the-leader approach to 
serpentine robot motion planning," ASCE Journal of Aerospace Engineering, vol. 12, no. 2, pp. 65-73, April 1999.

[8] J. W. Demmel, Applied Numerical Linear Algebra, SIAM, Philadelphia, PA, 1997.

[9] M. Erdmann, "On a representation of friction in configuration space," International Journal Of Robotics Research, vol. 13, no. 3, pp. 240-271, 1994.

[10] P. Fiorini and J. Burdick, "The development of hopping capabilities for small robots," Autonomous Robots, vol. 14, no. 2-3, pp. 239-254, May 2003.

[11] E. Hale, N. Schara, J. W. Burdick, and P. Fiorini, "A minimally actuated hopping rover for exploration of celestial bodies," in Proceedings of the IEEE International Conference On Robotics and Automation, San Francisco, CA, April 2000, pp. 420-7.

[12] S. Hirai and H. Asada, "Kinematics and statics of manipulation using the theory of polyhedral convex cones," International Journal Of Robotics Research, vol. 12, no. 5, pp. 434-447, 1993.

[13] J. Hodgins and M. H. Raibert, "Biped gymnastics," International Journal of Robotics Research, vol. 9, no. 2, pp. 115-132, 1990.

[14] H. P. Huang and N. H. McClamroch, "Time-optimal control for a robotic contour following problem," IEEE Journal of Robotics and Automation, vol. 4, no. 2, pp. 140-149, April 1988.

[15] T. Huynh, C. Lassez, and J-L. Lassez, "Practical issues on the projection of polyhedral sets," Ann. Math Artificial Intelligence, vol. 6, pp. 295-316, 1992.

[16] C. E. Lemke, "Recent results in complementarity problems," in Nonlinear Programming, J. B. Rosen et al., Ed., pp. 349-384. Academic Press, 1970.

[17] P. Lostedt, "Mechanical systems of rigid-bodies subject to unilateral constraints," SIAM Journal of Applied Mathematics, vol. 42, no. 2, pp. 281-296, April 1982.

[18] M. T. Mason, Mechanics of Robotic Manipulation, The MIT Press, first edition, 2001

[19] L. Matthies, Y. Xiong, R. Hogg, D. Zhu, Rankin A., B. Kennedy, M. Hebert, R. Maclachlan, C. Won, T. Frost, G. Sukhatme, M. McHenry, and S. Goldberg, "A portable, autonomous, urban reconnaissance robot," in Proceedings of The 6th International Conference on Intelligent Autonomous Systems, Venice, Italy, July 2000.

[20] M. D. P. Monteiro Marques, "Differential inclusions in nonsmooth mechanical problems: Shocks and dry friction.," Prog. Nonlinear Differential Equations Appl., vol. 9, 1993.

[21] J. Nakanishi, T. Fukuda, and D. E. Koditschek, "A brachiating robot controller," IEEE Transactions on Robotics and Automation, vol. 16, no. 2, pp. 109-123, 2000.

[22] Y. Or and E. Rimon, "Robust multiple-contact postures in a two-dimensional gravitational field," Tech. Rep., Technion University Dept. of Mechanical Engineering, 2003.

[23] J. S. Pang and J. C. Trinkle, "Stability characterizations of rigid body contact problems with coulomb friction," in Proc. IEEE Int. Conf. on Robotics and Automation, San Francisco, CA, April 2000, vol. 1, pp. 361-368.

[24] J. Peng, M. Anitescu, and S. Akella, "Optimal control of multiple robot systems with friction using mathematical programs with complementarity constraints," in Proc. IEEE Int. Conf. on Robotics and Automation, April 2004, vol. 5, pp. 5224-5231.

[25] F. Pfeiffer and C. Glocker, Multibody Dynamics with Unilateral Contacts, Wiley, first edition, 1996.

[26] D. Prattichizzo and A. Bicchi, "Dynamics analysis of mobility and graspability of general manipulation systems," IEEE Transactions on Robotics and Automation, vol. 14, no. 2, pp. 241-258, April 1998.

[27] M. Raibert, Legged Robots that Balance, MIT Press series in artificial intelligence. MIT Press, Boston, 1986.

[28] V. T. Rajan, R. Burridge, and T. Schwartz, "Dynamics of a rigid body in frictional contact with rigid walls," in Proc. IEEE Int. Conf. on Robotics and Automation, March 1987, vol. 4, pp. 671-677.

[29] U. Saranli, M. Buehler, and D. E. Koditschek, "RHex: A simple and highly mobile robot," International Journal of Robotics Research, vol. 20, no. 7, pp. 616-631, July 2001.

[30] U. Saranli, A. A. Rizzi, and D. E. Koditschek, "Model-based dynamic self-righting maneuvers for a hexapedal robot," International Journal of Robotics Research, vol. 23, no. 9, pp. 903-918, 2004.

[31] David E. Stewart, "Rigid-body dynamics with friction and impact," SIAM Review, vol. 42, no. 1, pp. 3-39, 2000.

[32] J. C. Trinkle, J. S. Pang, S. Sudarsky, and G. Lo, "On dynamic multi-rigid-body contact problems with Coulomb friction," Zeithschrift fur Angewandte Mathematik und Mechanik, vol. 77, pp. 267-279, 1997.

[33] E. Tunstel, "Evolution of autonomous self-righting behaviors for articulated nanorovers," in Proceedings of the 5th International Symposium on Artificial Intelligence, Robotics and Automation in Space, Noordwijk, The Netherlands, June 1999, pp. 341-6.

[34] R. J. Vanderbei, Linear Programming : Foundations and Extensions, Kluwer Academic, 2001. 